\title{
Conjugated linolenic fatty acids trigger ferroptosis in triple-negative breast cancer
}

Alexander Beatty ${ }^{1}$, Tanu Singh ${ }^{1}$, Yulia Y. Tyurina ${ }^{2,3}$, Emmanuelle Nicolas ${ }^{1}$, Kristen Maslar ${ }^{4}$, Yan Zhou ${ }^{1}$, Kathy Q. Cai ${ }^{1}$, Yinfei Tan ${ }^{1}$, Sebastian Doll ${ }^{5}$, Marcus Conrad ${ }^{5}$, Hülya Bayır ${ }^{2,3,6}$, Valerian E. Kagan ${ }^{2,3,7,8,9,10,}$ Ulrike Rennefahrt ${ }^{11}$, Jeffrey R. Peterson ${ }^{1 *}$

${ }^{1}$ Fox Chase Cancer Center, Philadelphia, Pennsylvania, USA

2 Department of Environmental and Occupational Health, University of Pittsburgh, Pittsburgh, Pennsylvania, USA

${ }^{3}$ Center for Free Radical and Antioxidant Health, University of Pittsburgh, Pittsburgh, Pennsylvania, USA,

4 Department of Biochemistry and Molecular Biology, Drexel University College of Medicine, Philadelphia, Pennsylvania, USA

${ }^{5}$ Institute of Developmental Genetics, Helmholtz Zentrum München, Neuherberg, Germany

${ }^{6}$ Department of Critical Care Medicine, Safar Center for Resuscitation Research, University of Pittsburgh, Pittsburgh, Pennsylvania, USA

${ }^{7}$ Department of Chemistry, University of Pittsburgh, Pittsburgh, Pennsylvania, USA,

${ }^{8}$ Department of Pharmacology and Chemical Biology, University of Pittsburgh, Pittsburgh, Pennsylvania, USA,

${ }^{9}$ Department of Radiation Oncology, University of Pittsburgh, Pittsburgh, Pennsylvania, USA,

10 Laboratory of Navigational Redox Lipidomics, IM Sechenov Moscow State Medical University, Moscow, Russia

${ }^{11}$ Metanomics Health GmbH, Berlin, Germany

* corresponding author, e-mail: Jeffrey.Peterson@fccc.edu 
Ferroptosis is a non-apoptotic form of cell death linked to the accumulation of reactive hydroperoxides generated by oxidation of polyunsaturated fatty acids (PUFAs) in membrane phospholipids. The therapeutic potential of promoting ferroptosis by enriching PUFAs in cancer cells is unknown. We found an association between elevated PUFA levels and vulnerability to ferroptosis in triple-negative breast cancer (TNBC) cells. A screen of PUFAs identified conjugated linolenic acids, including $\alpha$-eleostearate, as ferroptosis inducers. Three conjugated double bonds were required for ferroptotic activity although their positioning and stereochemistry were less significant. Mechanistically, $\alpha$-eleostearate differed from canonical ferroptosis inducers by a distinct dependence on acyl-CoA synthetase long-chain isoforms and by not altering glutathione or glutathione peroxidase 4 activity. Orally administered tung oil, naturally rich in $\alpha$-eleostearate, limited tumor growth and metastasis in an aggressive TNBC xenograft model. These results expand our understanding of ferroptotic cell death and highlight the anti-cancer potential of conjugated PUFAs.

Ferroptosis is a recently described form of iron-dependent, regulated cell death that is associated with elevated lipid hydroperoxides, a form of reactive oxygen species (ROS) ${ }^{1-6}$. Lipid hydroperoxides arise from enzymatic or spontaneous oxidation of membrane-associated polyunsaturated fatty acids (PUFAs). The central ferroptotic regulator is glutathione peroxidase 4 (GPX4), which is the only known peroxidase capable of efficiently reducing esterified, oxidized fatty acids into unreactive alcohols ${ }^{7}$. Small molecules that trigger ferroptosis identified thus far include agents that inhibit GPX4 directly, molecules that deplete the GPX4 cofactor glutathione, and compounds that oxidize iron, all of which lead to lipid hydroperoxide accumulation $^{1,8}$. Elevated lipid hydroperoxides lead to disruption of membrane architecture, production of reactive aldehydes, and ultimately cell death ${ }^{9}$. While the general mechanism of ferroptosis has been described, important details including metabolic features that render some cells susceptible to ferroptosis are not well understood. 
Recent studies have shown that cancer cells, including renal cell carcinoma, melanoma and glioblastoma are vulnerable to ferroptosis ${ }^{10-16}$. Furthermore therapy-resistant mesenchymal cancer cells exhibit a greater reliance on GPX4 activity for survival ${ }^{10,17}$. These findings have highlighted the therapeutic potential of pro-ferroptotic agents, but it remains unclear whether induction of ferroptosis can be used therapeutically to selectively kill cancer cells in vivo ${ }^{1}$. A major challenge to testing this hypothesis has been the absence of selective ferroptosis-inducing agents, such as GPX4 inhibitors, suitable for use in vivo ${ }^{18}$.

While suppressing the GPX4-mediated antioxidant response is one approach to induce ferroptosis, a complementary approach is to promote the production of lipid hydroperoxides by increasing the availability of their PUFA precursors. Hydroperoxides can spread by a free radical-mediated chain reaction leading to oxidation of adjacent PUFAs, and consequently higher PUFA levels could increase vulnerability to the propagation of lipid peroxides. Indeed, supplementation of cells with the PUFA arachidonic acid sensitizes them to ferroptosis triggered by GPX4 inhibitors ${ }^{19}$. Importantly, this approach to enhancing ferroptosis would exploit the propensity of cancer cells to scavenge fatty acids from their environment ${ }^{20}$. Here we report a vulnerability in triple-negative breast cancer (TNBC) to ferroptosis associated with the accumulation of PUFAs, and identified conjugated linolenic fatty acids as PUFAs that induce ferroptosis. Mechanistically, accumulation of PUFAs in TNBC triggered ferroptosis by a mode of action distinct from canonical ferroptosis inducers. We show that oral administration of tung nut oil, naturally rich in the conjugated PUFA $\alpha$-eleostearate, has anti-cancer activity in a xenograft model of TNBC. $\alpha$-Eleostearate metabolites could be detected in tumors of treated mice and was associated with expression of ferroptotic markers. These results introduce a distinct class of ferroptosis inducers and offer novel insights into the molecular basis of ferroptotic sensitivity. The tractability of these dietary, pro-ferroptotic fatty acids addresses the current lack of effective GPX4 inhibitors for use in vivo and suggests a novel opportunity to exploit a metabolic liability in an aggressive breast cancer subtype. 


\section{RESULTS}

\section{Glutathione depletion triggers ferroptosis in TNBC cell lines}

Survival of triple-negative breast cancer cells is dependent on the glutathione biosynthetic pathway to reduce ROS levels ${ }^{21}$. The source of ROS, however, has been unclear. Treatment with buthionine sulfoximine (BSO), an inhibitor of the rate-limiting enzyme of glutathione biosynthesis, triggers cell death in BT-549 cells within days of treatment (Fig. 1a). Glutathione depletion in some contexts induces cell death via ferroptosis ${ }^{22}$. Ferroptotic cell death is associated with increased lipid peroxidation and can be suppressed by lipophilic antioxidants like ferrostatin-1 (fer-1) and iron chelators ${ }^{1}$. Consistent with ferroptosis, cytotoxicity in BSO-treated BT-549 cells was blocked by fer-1 but not by an inhibitor of apoptotic cell death, the pan-caspase inhibitor Z-VAD-FMK (Figs. 1a, b). Similarly, iron chelators deferoxamine (DFO) and deferiprone suppressed BSO-mediated cell death (Fig. 1c, Supplementary Figure S1a). Furthermore, BSO treatment resulted in the accumulation of lipid peroxidation products and this was suppressed by fer-1 (Figs. 1d, e). Taken together, this demonstrates that glutathione depletion triggers ferroptosis in BT-549 cells and identifies lipid hydroperoxides as the lethal source of ROS underlying glutathione addiction in TNBC cells.

\section{Sensitivity to ferroptosis is associated with the accumulation of PUFAs}

Fer-1 suppressed BSO toxicity in half of the TNBC cell lines tested (Fig. 1f). These "ferroptotic" cell lines (red bars) were also generally more sensitive to ML162, a small molecule inhibitor of GPX4 23 (Welch's t-test, $\mathrm{p}=0.01$, Fig. 1g, Supplementary Figure S1b). Thus, ferroptosis is a common response to glutathione depletion in a subset of TNBC cells and is associated with a greater dependence on GPX4 activity. Notably, GPX4 transcripts were not differentially expressed between these two sets of cell lines (Supplementary Figure S1c), suggesting that ferroptotic vulnerability was independent of the level GPX4 expression. We used ANOVA of our prior metabolomic data for these same cell lines to compare levels of individual metabolites ${ }^{21}$ between TNBC cell lines susceptible to BSO-mediated ferroptosis and those that 
are not (as defined in Fig. 1f), and found that two of the three most significantly differentially expressed metabolites were linoleate (18:2)-substituted phosphatidylcholines, differing only in the identity of their other acyl chain $($ Fig. 1h, i). These membrane phospholipids were more enriched in ferroptotic compared to non-ferroptotic TNBC cell lines. Because PUFAs can be directly oxidized into lipid hydroperoxides, this finding suggested the possibility that elevated PUFA-containing phospholipids may increase vulnerability to ferroptosis and underlie these cells' greater dependence on GPX4 for survival (Fig. 1g). Consistent with this, recent studies implicate membrane phospholipids containing residues of arachidonic acid, and adrenic acid as promoters of ferroptosis ${ }^{19,24}$.

\section{The conjugated PUFA $\alpha$-eleostearic acid promotes cancer-selective ferroptosis}

We postulated that if PUFA levels are limiting for ferroptosis, elevating levels of specific PUFAs might further sensitize TNBC cells to ferroptosis. We tested a variety of PUFA species and identified the conjugated linolenic acid isomer $\alpha$-eleostearic acid [(9Z,11E,13E)-octadeca-9,11,13-trienoic acid; $\alpha \mathrm{ESA}]$ (Fig. 2a) as a fatty acid that enhanced cell death in combination with BSO (Fig. 2b). Unexpectedly, $\alpha$ ESA also triggered cell death as a single agent and this death was suppressed by fer-1 (Fig. 2b), the iron chelator deferoxamine (Fig. 2c) and by the lipophilic antioxidant vitamin E (Supplementary Figure S2a) and was associated with an increase in lipid peroxidation products that could be suppressed by fer-1 (Fig. 2d). Neither the pan-caspase inhibitor Z-VAD-FMK nor the necroptosis inhibitor nec-1s blocked $\alpha$ ESA-induced cell death (Supplementary Figure S2b, c). Together, these findings demonstrate that $\alpha$ ESA can induce ferroptosis as a single agent.

Interestingly, $\alpha \mathrm{ESA}$ is abundant in certain plants (e.g. bitter melon and the tung tree) ${ }^{25}$ and was previously shown to suppress the growth of breast cancer cells in a manner reversible by antioxidants, though the exact mechanism was unclear ${ }^{26}$. The non-transformed MCF-10A breast epithelial cell line was resistant to death by $\alpha \mathrm{ESA}$ (Fig. 2e). By contrast, all eight tested TNBC cell lines were susceptible to cell 
death by $\alpha E S A$, including cell lines that did not undergo ferroptosis in response to glutathione depletion

(Fig. 1f), and in all cases death could be prevented by fer-1 (Figs. 2b, $\mathbf{f}$ and not shown). Time lapse phase contrast microscopy of $\alpha$ ESA-treated BT549 cells (Supplementary Video 1) showed that during the latter part of 24 hours of treatment, otherwise healthy appearing cells undergo a sudden death, morphologically similar to cell death by the canonical ferroptosis inducer ML162 (Supplementary Video 2) and distinct from apoptotic death induced by staurosporine (Supplementary Video 3). Cell death by $\alpha$ ESA was associated with a large membrane protrusion that ultimately ruptured, accompanied by a cessation of cytoplasmic motility.

\section{Structure-activity analysis of conjugated PUFAs}

$\alpha \mathrm{ESA}$ is a naturally occurring fatty acid with no structural similarity to known ferroptosis inducers. To characterize structural features of $\alpha$ ESA required for ferroptosis, we screened structurally related conjugated and unconjugated PUFAs for death induction in BT549 cells. $\alpha$ ESA possesses conjugated double bonds at carbons 9, 11, and 13 with cis, trans, trans stereochemistry, respectively (Fig. 2g). We examined cis-trans stereoisomers of $\alpha \mathrm{ESA}$ and found that isomerization at position 9 ( $\beta$-eleostearic acid) or at both 9 and 13 positions (catalpic acid) retained cell death activity while isomerization at position 13 alone (punicic acid) led to an $\sim$-fold reduction in potency. Jacaric acid (conjugated 18:3, cis-8, trans-10, cis-12) showed the most potent ability to kill cells $(1.8 \mu \mathrm{M} \mathrm{IC} 50)$, demonstrating that shifting the positioning of the double bonds while maintaining the sequential cis, trans, cis stereochemistry of punicic acid, did not disrupt activity. $\alpha$-Calendic acid (conjugated 18:3, trans-8, trans-10, cis-12), a stereoisomer of jacaric acid, was similarly potent. Thus, cell killing is not strictly dependent on the precise positioning or stereochemistry of double bonds within the fatty acid. A conjugated linoleic acid (18:2), which shares similar position and stereochemistry of its two double bonds with $\alpha \mathrm{ESA}$, was a poor inducer of cell death, however, suggesting that third conjugated double bond is required for its activity. Furthermore, all tested non-conjugated PUFAs (e.g. docosahexaenoic, linoleic, and linolenic acids) were less potent single-agent inducers of cell death 
(Supplementary Figure S2d) including arachidonic acid (20:4), which was of interest because it enhances ferroptosis in some contexts ${ }^{19,27}$. Cell killing by arachidonic acid could not be rescued by fer-1 (Supplementary Figure S2e). By contrast, jacaric- and catalpic acid-induced death, like that caused by aESA, was suppressed by fer-1 (Supplementary Figure S2f). Similar results were found in BT-549 cells (Supplementary Figure S2g), demonstrating the conserved ability of diverse conjugated 18:3 fatty acids to trigger ferroptosis.

\section{aESA is incorporated into diverse cellular lipids}

$\alpha \mathrm{ESA}$, unlike canonical ferroptotic agents, is a fatty acid that could potentially be incorporated as an acyl chain in cellular lipids. To examine how $\alpha \mathrm{ESA}$ is metabolized, we conducted a mass spectrometrybased lipidomic analysis of MDA-MB-231 cells that were untreated, $\alpha$ ESA-treated, fer-1-treated, or treated with both $\alpha \mathrm{ESA}$ and fer-1 for three hours. While 18:3-containing lipids were extremely rare in untreated cells, $\alpha$ ESA-treatment was associated with incorporation of 18:3 acyl chains, into a diverse array of lipid species including phospholipids, cholesterol esters and storage lipids and fer-1 did not dramatically alter the spectrum of lipid classes that incorporated 18:3 acyl chains (Fig. 2h; detailed results are presented in Supplementary Table 1). The abundance of di- and triacylglycerol lipids increased $\sim 2$-fold in $\alpha$ ESA- and $\alpha \mathrm{ESA}+$ fer-1-treated cells compared to untreated controls, suggesting that a portion of $\alpha \mathrm{ESA}$ is incorporated into storage lipids (Fig. 2i). All lipid classes significantly altered by $\alpha \mathrm{ESA}$ treatment were similarly altered by $\alpha \mathrm{ESA}$ in the presence of fer-1 (Supplementary Table 1). $\alpha \mathrm{ESA}$-induced lipidomic changes were not simply downstream consequences of ferroptosis since the effects were not replicated by ML162 treatment (Fig. 2h, i). Together these results demonstrate that $\alpha$ ESA is incorporated into cellular fatty acid pools in a largely fer-1-insensitive manner. 


\section{Conjugated PUFAs exhibit anti-cancer activity and promote expression of ferroptotic markers in vivo}

We used tung oil as an inexpensive and rich source of $\alpha$ ESA $(80 \%$ of all fatty acids in tung oil are $\alpha \mathrm{ESA}^{28}$ ) to assess whether its anti-TNBC activity could be recapitulated in the aggressive MDA-MB-231 orthotopic xenograft model. Once tumors reached $\sim 75 \mathrm{~mm}^{3}$, mice were treated by oral gavage five times per week with $100 \mu 1$ tung oil. Control mice received high-oleic (18:1) safflower oil. Body weights of tung oil-treated mice did not differ significantly from controls over the treatment period (not shown). However, tung oil significantly suppressed tumor growth (Fig. 3a) and endpoint tumor weight (Fig. 3b) and also substantially reduced lung metastatic invasion compared to controls (Figs. 3c, d). Immunohistochemistry revealed a 10\% decrease in Ki67-positive cells in tumors from tung-treated mice compared to controls and a modest, though statistically significant increase in tumor cleaved caspase 3-positive cells, a marker of apoptosis (from 2\% to 5\%) (Supplementary Figure S3a, b).

To provide evidence that the reduction in tumor growth is associated with tumor exposure to $\alpha \mathrm{ESA}$, we conducted a lipidomic analysis of $\alpha$ ESA-treated and control tumors. The two most differentially expressed lipids between the tumor groups were diacylglycerol (34:3) and triacylglycerol (52:7), which were increased in tung oil-treated tumors by $30 \%$ and $60 \%$, respectively (Fig. 3e). The masses of these lipids are consistent with at least one linolenate moiety, although their precise acyl chain composition was not determined. Notably, the expression of these two lipids was also increased in cells treated with $\alpha$ ESA in vitro, compared to untreated cells (Fig. 3e). Our findings identify the neutral lipids diacylglycerol (34:3) and triacylglycerol (52:7) as potential biomarkers of tumor exposure to $\alpha \mathrm{ESA}$. Further, they suggest that circulating $\alpha \mathrm{ESA}$, released from tung oil, is taken up by TNBC tumor cells and incorporated into lipids.

Next, we assessed whether tumors in $\alpha$ ESA-treated mice expressed RNA markers of ferroptosis. We conducted RNA sequencing of three biological replicates each of tumors from treated and control mice and compared the differentially expressed genes with a separate in vitro experiment using cultured MDA- 
MB-231 cells treated with either $\alpha$ ESA, the GPX4 inhibitor ML162, or corresponding vehicle controls. Using a false discovery rate of 5\% and a filter of $>2$-fold change in RNA expression level, 124 genes were altered in tung oil-treated tumors compared to safflower oil-treated controls (Fig. 3f). Significantly, 89 genes $(89 / 122,73 \%)$ altered in tung oil-treated tumors were also altered in $\alpha$ ESA-treated cells in culture. Fisher's exact test showed extremely significant overlap between these gene sets $\left(p=3.2 \times 10^{-246}\right)$ and all 89 overlapping genes were altered in the same direction (Supplementary Table 2). This is consistent with our hypothesis that the inhibition of tumor growth in vivo is related to the cell death caused by $\alpha$ ESA in vitro. Importantly, there was also a highly significant overlap in genes altered by tung oil in vivo, $\alpha \mathrm{ESA}$ in vitro, and by in vitro treatment with ML162 (Fig. 3f and Supplementary Table 2). Fisher's exact test between any two gene sets demonstrated $\mathrm{p}$-values of $<7.2 \times 10^{-61}$. Furthermore, all of the shared genes were altered in the same direction. These results demonstrate highly concordant gene signatures between tung oil treatment and $\alpha \mathrm{ESA}$ treatment, consistent with the hypothesis that tumors in tung oil-treated mice are exposed to $\alpha$ ESA. Furthermore, the highly significant overlap in the signatures of $\alpha$ ESA- and ML162-treated cells provide strong evidence that both treatments trigger a similar response.

All three treatments altered a common set of 23 genes (Fig. $3 f$ and Supplementary Table 2) including genes previously identified as increased during ferroptosis including RHOB, SLC2A3, DDIT4 and HMOX1 15,29. In addition, the previously reported ferroptosis marker CHAC1 ${ }^{29}$ was commonly upregulated in both tung-oil treated tumors and $\alpha$ ESA-treated cells. 66 genes were altered by both $\alpha$ ESA and tung oil but not ML162 (Supplementary Table 2), suggesting a potential source of biomarkers for $\alpha$ ESA-induced ferroptosis.

\section{Ferroptosis induction by $\alpha$ ESA is not mediated by GPX4 inhibition}

Next, we sought mechanistic insights into ferroptosis induction by $\alpha$ ESA. One possibility is that $\alpha$ ESA could promote the formation of lipid hydroperoxides by inhibiting GPX4 either directly or indirectly. 
For example, ML162 and the structurally distinct GPX4 inhibitor RSL3 directly bind and inactivate GPX4 while BSO and erastin indirectly inhibit GPX4 by starving GPX4 of its essential cofactor glutathione ${ }^{1}$. While BSO treatment suppressed glutathione levels, treatment of cells with aESA for 24 hours did not significantly affect total cellular glutathione levels despite decreasing cell viability (Fig. 4a), suggesting that $\alpha$ ESA does not trigger ferroptosis by depleting glutathione.

Direct GPX4 inhibitors have been shown to reduce GPX4 protein levels ${ }^{19}$. We confirmed this for ML162 and RSL3 but found that $\alpha$ ESA or the negative control erastin had no effect on GPX4 levels (Fig. 4b), suggesting that $\alpha$ ESA does not inhibit GPX4 activity in a similar way. As an alternative approach to determine whether $\alpha \mathrm{ESA}$ inhibits GPX4 either directly or indirectly, we compared the kinetics of ferroptosis induction in TNBC cells treated with $\alpha$ ESA, ML162 and RSL3 alone and in combination. The combination of both GPX4 inhibitors at $500 \mathrm{nM}$ triggered fer-1-suppressible cell death with similar kinetics to either GPX4 inhibitor alone, indicating this dose of inhibitor was sufficient to achieve near maximal GPX4 inhibition (Fig 4c). In contrast, cell viability was lost significantly more rapidly when cells were co-treated with $500 \mathrm{nM}$ ML162 and $50 \mu \mathrm{M} \alpha \mathrm{ESA}$, a dose of $\alpha \mathrm{ESA}$ that took longer to induce cell death than either of the GPX4 inhibitors alone. Consistent with ferroptosis, cell death triggered by the combination of ML162 and $\alpha$ ESA could be fully rescued by fer-1 (Fig 4c). The ability of $\alpha$ ESA to further enhance the kinetics of cell death under conditions of maximal GPX4 inhibition suggests that $\alpha$ ESA and GPX4 inhibitors trigger ferroptosis by distinct mechanisms.

\section{aESA-induced ferroptosis is lipoxygenase and ACSL1-dependent}

Ferroptosis is intimately associated with PUFA peroxidation ${ }^{30,31}$. Lipoxygenases have been reported to mediate peroxidation in ferroptosis induced by GPX4 inhibition ${ }^{24,27}$ but non-enzymatic lipid oxidation by Fenton chemistry may also contribute to this process ${ }^{18,32}$. To assess lipoxygenase contributions to $\alpha$ ESA-induced ferroptosis we systematically knocked down lipoxygenase isoforms by RNA interference 
in two TNBC cell lines. ALOXE3 knockdown most significantly suppressed $\alpha$ ESA toxicity in both cell lines (Fig. 4d, e), demonstrating a role for this lipoxygenase isoform in $\alpha E S A$-induced death. This finding does not, however, rule out a potential role for non-enzymatic oxidation, since $\alpha \mathrm{ESA}$ undergoes spontaneous oxidation more rapidly than other unconjugated and conjugated linolenic acids ${ }^{33,34}$.

The first step in esterification of fatty acids into cellular lipids is their conjugation to CoA, catalyzed by enzymes of the acyl-CoA synthetase family. There are five long chain-specific isoforms (ACSLs) and loss of ACSL4 significantly protects cells from ferroptosis induced by direct or indirect inhibition of GPX4 (Fig. 4f; ${ }^{16,35,36}$ ). $\alpha$ ESA, however, was similarly toxic to ACSL4-deficient Pfal mouse embryonic fibroblast cells and control parental cells (Fig. 4g), suggesting a difference in the mechanism of action of $\alpha$ ESA and canonical ferroptosis inducers. $\alpha$ ESA-induced cell death was rescued by fer-1 treatment in both cell lines, consistent with ferroptosis (Supplementary Figure S4a). To determine if any ACSL isoforms contribute to ferroptosis induced by $\alpha \mathrm{ESA}$, we individually knocked down expression of each of the five ACSL isoforms in BT549 and MDA-MB-231 cells by RNAi. Knockdown of individual ACSL isoforms expressed in each cell line was confirmed by quantitative PCR (Supplementary Figures S4b, c). As expected, ML162 toxicity was most effectively suppressed by ACSL4 depletion in both cell lines. $\alpha$ ESA toxicity, however, was most significantly suppressed by knockdown of ACSL1 (Figs. 4h, i). Thus, $\alpha$ ESA differs in its ACSL isoform dependence compared to a conventional ferroptosis inducer, further supporting a mechanism distinct from canonical ferroptosis inducers.

\section{aESA triggers ACSL1-dependent increases in lipid hydroperoxides}

As ferroptosis is associated with an accumulation of oxidized phospholipids, we next used liquid chromatography-mass spectrometry (LC-MS) to ask if $\alpha$ ESA treatment impacts phospholipid oxidation. We quantified 141 mono-, di-, and tri- oxygenated lipids across seven lipid classes in BT-549 cells and found the abundance of oxidized lipids was significantly increased in cells treated with $\alpha$ ESA $(p=0.02)$ 
(Fig. 5a). Overall, the amounts of 51 (36\%) lipid species were significantly changed and a majority of those were increased $(41 / 51,80 \%)$. The classes with both the highest number and frequency of increased oxidized species were cardiolipins (17/36 47\%) and phosphatidylethanolamines (18/40 45\%) and included two dioxygenated phosphatidylethanolamine species containing arachidonate residues (Fig. 5b) that have been previously identified as ferroptotic signals in cells treated with the GPX4 inhibitor RSL3 ${ }^{19}$. These results are consistent with our observation of increased lipid peroxidation products in $\alpha$ ESA-treated cells (Fig. 2d) and suggest that oxidized PE and CL phospholipids may contribute to ferroptosis triggered by $\alpha$ ESA.

One possible explanation for the ACSL1-dependence of $\alpha$ ESA-induced ferroptosis is that ACSL1 mediates the incorporation of $\alpha$ ESA into cellular lipids. To test this, we sought to quantify the presence of 18:3 fatty acids in diverse lipid species in $\alpha$ ESA- and control-treated cells in which ACSL1 expression was silenced (Fig. 5c). Total acyl chain length and double bond count were characterized for each lipid species containing two acyl chains (excludes cardolipins) and we focused on 36:6 lipids because this composition is consistent with two $\alpha$ ESA acyl chains. Validating this approach, $\alpha$ ESA treatment led to a 13 -fold increase in total 36:6 lipids (Fig. 5d), which were distributed across all examined lipid classes (Fig. 5e). Silencing ACSL1 did not affect overall 36:6 lipid levels in aESA-treated cells, demonstrating that ACSL1 is not essential for $\alpha E S A$ esterification, although some individual classes exhibited significantly decreased 36:6 lipids. An alternative hypothesis is that ACSL1 depletion suppresses $\alpha$ ESA-induced ferroptosis by lessening the accumulation of oxidized lipid species. We quantified oxygenated lipids in $\alpha$ ESA-treated cells in which ACSL1 was knocked down (Fig. 5f). Of the oxygenated lipids that were significantly increased following incubation with $\alpha \mathrm{ESA}, 10(10 / 41,24 \%)$ were responsive to ACSL1 depletion and all were decreased (detailed in Supplementary Figure S5), consistent with the idea that decreasing ACSL1 levels may reduce the accrual of specific peroxidized phospholipids that induce ferroptosis in $\alpha E S A$-treated cells. Indeed, we found that some, but not all, $\alpha$ ESA-induced, di-oxygenated PE lipids were decreased on ACSL1-silencing (Fig. 5b). 


\section{Small-molecule enhancers of $\alpha$ ESA-induced ferroptosis implicate phospholipase A2 as a suppressor}

\section{of ferroptosis}

Since $\alpha$ ESA causes an accumulation of lipid hydroperoxides (Fig. 5c) without decreasing GPX4 (Fig. 4b), its toxicity might be enhanced in combination with GPX4 inhibitors. Consistent with this, low doses of BSO, which on their own do not affect cellular viability, enhanced $\alpha$ ESA toxicity in vitro (Fig. 2b). Similarly, treatment of mice with BSO at a dose that does not affect tumor growth on its own ${ }^{21}$, enhanced the anti-tumor activity of tung oil in MDA-MB-231 orthotopic xenografts (Fig. 6a). We likewise found that subtoxic doses of $\alpha \mathrm{ESA}$ enhanced cell killing by the GPX4 inhibitors ML162 and RSL3 in multiple cell lines (Fig. 6b and Supplementary Figure S6). Taken together, our observations are consistent with $\alpha$ ESA promoting an increase in lipid hydroperoxides that is opposed by, but ultimately overwhelms GPX4 activity. In addition, they suggest that combining GPX4 inhibitors, should these become clinically viable, with $\alpha$ ESA may enhance the anti-tumor activity of $\alpha \mathrm{ESA}$.

Finally, we sought to identify other metabolic pathways that might modulate ferroptosis in TNBC cells. We conducted a multi-dose screen of 126 known small molecule inhibitors of diverse metabolic pathways for their ability to modify the toxicity of glutathione depletion in MDA-MB-231 cells. Both enhancers and suppressors of BSO toxicity were identified, including previously reported enhancers of ferroptosis, auranofin ${ }^{37,22}$, and sorafenib ${ }^{29}$ (Fig. 6c). Among the most potent enhancers was the multitargeting compound RHC-80267. As a single agent, RHC-80267 only weakly induced cell death (Fig. 6d, e). In combination with subtoxic BSO, however, RHC-80267 induced fer-1 suppressible cell death, demonstrating a synthetic lethal interaction between these two compounds (Fig. 6e). In untransformed MCF-10A cells BSO did not enhance RHC-80267 toxicity (Fig. 6d). RHC-80267-induced cell death was also enhanced by $\alpha \mathrm{ESA}$ in multiple cell types and could be fully rescued by fer-1 (Fig. 6e; other cell lines not shown). Thus, RHC-80267 is a selective enhancer of ferroptosis triggered by either $\alpha \mathrm{ESA}$ or canonical ferroptosis inducers. 
One target of RHC-80267 is diacylglycerol lipase ${ }^{22}$ but knock down of DAGL1 and DAGL2 isoforms individually and together did not affect $\alpha$ ESA potency (not shown). RHC-80267 also exhibits inhibitory activity against phospholipase A2 (PLA2) ${ }^{38}$. To probe a potential role for PLA2 isoforms we used three additional, structurally unrelated PLA2 inhibitors, each of which enhanced ferroptotic death by $\alpha$ ESA (Figs. 6f). In every case, cell death was suppressed by fer-1. These results point to a key role for PLA2 in protecting against both BSO- and $\alpha$ ESA-induced ferroptosis. PLA2 isoforms play a role in phospholipid repair by liberating oxidatively damaged fatty acids from membrane phospholipids ${ }^{39}$. Thus, inhibition of PLA2 may promote $\alpha$ ESA-induced ferroptosis by allowing the accumulation of oxidized fatty acids in membrane phospholipids.

\section{DISCUSSION}

Cancer cells, particularly drug-resistant and metastatic cells are vulnerable to oxidative stress and depend on antioxidant pathways for their survival $10,40-45$. This metabolic liability could potentially be exploited by two complementary therapeutic approaches, inhibiting anti-oxidant survival pathways or promoting oxidative stress. Consistent with the first strategy, we and others have demonstrated a dependence on glutathione-based anti-oxidant pathways in breast cancer ${ }^{21,42,46,47}$. Here, we demonstrate the feasibility of the second strategy, enhancing oxidative stress using conjugated linolenic acids to trigger the production of reactive hydroperoxides, a key mediator of ferroptosis.

GPX4 acts as a central regulator of ferroptosis by preventing the accumulation of phospholipid hydroperoxides. Existing small-molecule inducers of ferroptosis directly or indirectly inhibit GPX4 activity, leading to the accumulation of lipid peroxides produced endogenously. We show here that conjugated linolenic fatty acids provide a distinct mechanism to initiate ferroptosis in which these oxidizable fatty acids increase the production of lipid peroxides that overwhelm GPX4 activity. 
Furthermore, the combination of these two approaches robustly enhances cell death both in vitro and in vivo

(Fig. 4c, Figs. 6a, b). Supporting a distinct mechanism of action, the pro-ferroptotic activity of $\alpha$ ESA relies on a subset of ACSL and lipoxygenase isoforms that is at least partially distinct from those exploited by canonical ferroptosis inducers. Despite these mechanistic differences, $\alpha$ ESA and conventional ferroptosis inducers both lead ultimately to an increase in lipid peroxides (Figs. 1e, 2d) and induce a similar transcriptional response (Fig. 3f), suggesting a convergent mechanism of lethality. While GPX4 inhibition allows the accumulation of endogenous lipid hydroperoxides, $\alpha$ ESA is incorporated into membrane lipids, increasing levels of oxidizable PUFAs, and enhancing overall lipid oxygenation. GPX4 deletion in adult mice leads to acute renal failure and death ${ }^{48}$, suggesting that the therapeutic window for clinical GPX4 inhibitors, should they become available, may be narrow. By contrast, tung oil and $\alpha$ ESA (unpublished data) appear to be well tolerated in mice. Thus, these findings expand our understanding of the pathways that regulate ferroptosis and provide a new approach for exploiting ferroptosis therapeutically.

Our metabolic inhibitor screen uncovered a potential role for PLA2 in preventing ferroptosis (Fig. 6), consistent with a recent study that identified secreted PLA2 as a suppressor of lipotoxic stress in breast cancer cells ${ }^{49}$. Inhibitors of PLA2 potentiate ferroptosis by both $\alpha$ ESA (Fig. 6f) and canonical ferroptosis inducers (Fig. 6d), possibly by preventing oxidatively damaged fatty acyl chains from being liberated from membrane lipids ${ }^{39}$. This finding suggests that PLA2 inhibitors might enhance the therapeutic efficacy of conjugated linolenic acids or canonical ferroptosis inducers in cancer. PLA2 isoforms constitute a large family of enzymes with potential functional redundancy, making it challenging to identify whether specific isoforms protect against ferroptosis. Nevertheless, this finding supports an emerging model in which dynamic remodeling of membrane fatty acids can modulate cellular vulnerability to PUFA oxidation ${ }^{50}$.

There are two major limitations to translating ferroptosis into a therapeutic modality, the lack of orally available ferroptosis inducers and the absence of robust biomarkers of ferroptosis for use in vivo. We 
discovered that tung oil, a naturally rich source of $\alpha$ ESA, suppresses xenograft tumor growth and metastasis and provide evidence that this activity is mediated by ferroptosis. These results identify tung oil as an inexpensive and bioavailable agent potentially suitable for clinical ferroptosis induction. Moreover, its lack of toxicity and positive interactions with other pro-ferroptotic agents, reported here, suggest opportunities for combination therapy. Finally, we identified a set of genes whose expression is altered in cells undergoing $\alpha$ ESA- and tung oil-induced ferroptosis as potential biomarkers of ferroptosis and a source of candidate genes that may contribute to this cell death mechanism.

\section{ACKNOWLEDGEMENTS}

This work was supported in part by NIH GM083025, U19AI068021, HL114453-06, CA165065-06, NS076511, the PA Breast Cancer Coalition, the Fifth District AHEPA Cancer Research Foundation, the Spurlino Family Foundation, the Rita Hollman Foundation, the Eileen Stein Jacoby Fund, and Translation Research and In Vino Vita awards from Fox Chase Cancer Center. This work was supported by NIH CORE Grant P30 CA006927. We thank Drs. S. Balachandran and J. Karanicolas for comments on the manuscript. 


\section{REFERENCES}

1. Cao, J.Y. \& Dixon, S.J. Mechanisms of ferroptosis. Cell Mol Life Sci 73, 2195-209 (2016).

2. Xie, Y. et al. Ferroptosis: process and function. Cell Death Differ 23, 369-79 (2016).

3. Yang, W.S. \& Stockwell, B.R. Ferroptosis: Death by Lipid Peroxidation. Trends Cell Biol 26, 16576 (2016).

4. Stockwell, B.R. et al. Ferroptosis: A Regulated Cell Death Nexus Linking Metabolism, Redox Biology, and Disease. Cell 171, 273-285 (2017).

5. Friedmann-Angeli, J.P., Miyamoto, S. \& Schulze, A. Ferroptosis: the greasy side of cell death. Chem Res Toxicol (2019).

6. Conrad, M. et al. Regulation of lipid peroxidation and ferroptosis in diverse species. Genes Dev 32, 602-619 (2018).

7. Conrad, M. \& Friedmann Angeli, J.P. Glutathione peroxidase 4 (Gpx4) and ferroptosis: what's so special about it? Mol Cell Oncol 2, e995047 (2015).

8. Gaschler, M.M. et al. FINO2 initiates ferroptosis through GPX4 inactivation and iron oxidation. Nat Chem Biol 14, 507-515 (2018).

9. Agmon, E., Solon, J., Bassereau, P. \& Stockwell, B.R. Modeling the effects of lipid peroxidation during ferroptosis on membrane properties. Sci Rep 8, 5155 (2018).

10. Viswanathan, V.S. et al. Dependency of a therapy-resistant state of cancer cells on a lipid peroxidase pathway. Nature 547, 453-457 (2017).

11. Kyani, A. et al. Discovery and Mechanistic Elucidation of a Class of Protein Disulfide Isomerase Inhibitors for the Treatment of Glioblastoma. ChemMedChem 13, 164-177 (2018).

12. Buccarelli, M. et al. Inhibition of autophagy increases susceptibility of glioblastoma stem cells to temozolomide by igniting ferroptosis. Cell Death Dis 9, 841 (2018).

13. Chen, L. et al. Erastin sensitizes glioblastoma cells to temozolomide by restraining xCT and cystathionine-gamma-lyase function. Oncol Rep 33, 1465-74 (2015). 
14. Lu, B. et al. The Role of Ferroptosis in Cancer Development and Treatment Response. Front Pharmacol 8, 992 (2017).

15. Hassannia, B. et al. Nano-targeted induction of dual ferroptotic mechanisms eradicates high-risk neuroblastoma. J Clin Invest 128, 3341-3355 (2018).

16. Doll, S. et al. ACSL4 dictates ferroptosis sensitivity by shaping cellular lipid composition. Nat Chem Biol (2017).

17. Hangauer, M.J. et al. Drug-tolerant persister cancer cells are vulnerable to GPX4 inhibition. Nature 551, 247-250 (2017).

18. Feng, H. \& Stockwell, B.R. Unsolved mysteries: How does lipid peroxidation cause ferroptosis? PLoS Biol 16, e2006203 (2018).

19. Kagan, V.E. et al. Oxidized arachidonic and adrenic PEs navigate cells to ferroptosis. Nat Chem Biol (2017).

20. Kamphorst, J.J. et al. Hypoxic and Ras-transformed cells support growth by scavenging unsaturated fatty acids from lysophospholipids. Proc Natl Acad Sci U S A 110, 8882-7 (2013).

21. Beatty, A. et al. Metabolite Profiling Reveals the Glutathione Biosynthetic Pathway as a Therapeutic Target in Triple-Negative Breast Cancer. Mol Cancer Ther 17, 264-275 (2018).

22. Dixon, S.J. et al. Ferroptosis: an iron-dependent form of nonapoptotic cell death. Cell 149, 1060-72 (2012).

23. Weiwer, M. et al. Development of small-molecule probes that selectively kill cells induced to express mutant RAS. Bioorg Med Chem Lett 22, 1822-6 (2012).

24. Yang, W.S. et al. Peroxidation of polyunsaturated fatty acids by lipoxygenases drives ferroptosis. Proc Natl Acad Sci US A 113, E4966-75 (2016).

25. Cui, P. et al. Tung Tree (Vernicia fordii, Hemsl.) Genome and Transcriptome Sequencing Reveals Co-Ordinate Up-Regulation of Fatty Acid beta-Oxidation and Triacylglycerol Biosynthesis 
Pathways During Eleostearic Acid Accumulation in Seeds. Plant Cell Physiol 59, 1990-2003 (2018).

26. Grossmann, M.E. et al. Eleostearic Acid inhibits breast cancer proliferation by means of an oxidation-dependent mechanism. Cancer Prev Res (Phila) 2, 879-86 (2009).

27. Seiler, A. et al. Glutathione peroxidase 4 senses and translates oxidative stress into 12/15lipoxygenase dependent- and AIF-mediated cell death. Cell Metab 8, 237-48 (2008).

28. Dyer, J.M. et al. Molecular analysis of a bifunctional fatty acid conjugase/desaturase from tung. Implications for the evolution of plant fatty acid diversity. Plant Physiol 130, 2027-38 (2002).

29. Dixon, S.J. et al. Pharmacological inhibition of cystine-glutamate exchange induces endoplasmic reticulum stress and ferroptosis. Elife 3, e02523 (2014).

30. Zilka, O. et al. On the Mechanism of Cytoprotection by Ferrostatin-1 and Liproxstatin-1 and the Role of Lipid Peroxidation in Ferroptotic Cell Death. ACS Cent Sci 3, 232-243 (2017).

31. Gaschler, M.M. \& Stockwell, B.R. Lipid peroxidation in cell death. Biochem Biophys Res Commun 482, 419-425 (2017).

32. Shah, R., Shchepinov, M.S. \& Pratt, D.A. Resolving the Role of Lipoxygenases in the Initiation and Execution of Ferroptosis. ACS Cent Sci 4, 387-396 (2018).

33. Myers, J.E., Kass, J.P. \& Burr, G.O. Comparative rates of oxidation of isomeric linolenic acids and their esters. Oil \& Soap 18, 107-109 (1941).

34. Tsuzuki, T. et al. Oxidation rate of conjugated linoleic acid and conjugated linolenic acid is slowed by triacylglycerol esterification and alpha-tocopherol. Lipids 39, 475-80 (2004).

35. Dixon, S.J. et al. Human Haploid Cell Genetics Reveals Roles for Lipid Metabolism Genes in Nonapoptotic Cell Death. ACS Chem Biol 10, 1604-9 (2015).

36. Yuan, H., Li, X., Zhang, X., Kang, R. \& Tang, D. Identification of ACSL4 as a biomarker and contributor of ferroptosis. Biochem Biophys Res Commun 478, 1338-43 (2016). 
37. Poursaitidis, I. et al. Oncogene-Selective Sensitivity to Synchronous Cell Death following Modulation of the Amino Acid Nutrient Cystine. Cell Rep 18, 2547-2556 (2017).

38. Hoover, H.S., Blankman, J.L., Niessen, S. \& Cravatt, B.F. Selectivity of inhibitors of endocannabinoid biosynthesis evaluated by activity-based protein profiling. Bioorg Med Chem Lett 18, 5838-41 (2008).

39. van Kuijk, F.J.G.M., Sevanian, A., Handelman, G.J. \& Dratz, E.A. A new role for phospholipase A2: protection of membranes from lipid peroxidation damage. Trends in Biochemical Sciences $\mathbf{1 2}$, 31-34 (1987).

40. Alvarez, S.W. et al. NFS1 undergoes positive selection in lung tumours and protects cells from ferroptosis. Nature 551, 639-643 (2017).

41. Gorrini, C., Harris, I.S. \& Mak, T.W. Modulation of oxidative stress as an anticancer strategy. Nat Rev Drug Discov 12, 931-47 (2013).

42. Luo, M. et al. Targeting Breast Cancer Stem Cell State Equilibrium through Modulation of Redox Signaling. Cell Metab 28, 69-86 e6 (2018).

43. Mai, T.T. et al. Salinomycin kills cancer stem cells by sequestering iron in lysosomes. Nat Chem 9, 1025-1033 (2017).

44. Piskounova, E. et al. Oxidative stress inhibits distant metastasis by human melanoma cells. Nature 527, 186-91 (2015).

45. Rojo de la Vega, M., Chapman, E. \& Zhang, D.D. NRF2 and the Hallmarks of Cancer. Cancer Cell 34, 21-43 (2018).

46. Harris, I.S. et al. Glutathione and thioredoxin antioxidant pathways synergize to drive cancer initiation and progression. Cancer Cell 27, 211-22 (2015).

47. Lien, E.C. et al. Glutathione biosynthesis is a metabolic vulnerability in PI(3)K/Akt-driven breast cancer. Nat Cell Biol 18, 572-8 (2016). 
48. Friedmann Angeli, J.P. et al. Inactivation of the ferroptosis regulator Gpx4 triggers acute renal failure in mice. Nat Cell Biol 16, 1180-91 (2014).

49. Jarc, E. et al. Lipid droplets induced by secreted phospholipase A2 and unsaturated fatty acids protect breast cancer cells from nutrient and lipotoxic stress. Biochim Biophys Acta Mol Cell Biol Lipids 1863, 247-265 (2018).

50. Magtanong, L. et al. Exogenous monounsaturated fatty acids promote a ferroptosis-resistant cell state. Cell Chem Biol 26, 1-13 (2019). 


\section{METHODS}

\section{Cell lines}

MDA-MB-468 cells were obtained by the Cell Culture Facility at Fox Chase Cancer Center from ATCC as part of the NCI-60 panel. All other TNBC cell lines and the MCF10A cell line were obtained directly from the American Type Culture Collection (ATCC, Manassas, VA 20110, USA). The suppliers routinely authenticate the cell lines by short tandem repeat profiling though only MDA-MB-231 cell lines were authenticated by our laboratory (April 2018). All cell lines were amplified and frozen within 2 months of receipt. TNBC cell lines were cultured in RPMI-1640, 10\% heat inactivated fetal bovine serum, $2 \mathrm{mM}$ supplemental glutamine, and $100 \mu \mathrm{g} / \mathrm{mL}$ penicillin/streptomycin with the exception of BT-20, which was cultured in MEM with the same supplements. MCF-10A cells were cultured in high calcium medium with 5\% horse serum as described ${ }^{51}$. Mouse embryonic fibroblast (Pfa1) Acsl4-deficient cells were described previously ${ }^{16}$. Cells were cultured in a humidified incubator at $37^{\circ} \mathrm{C}$ with $5 \% \mathrm{CO}_{2}$. Cell lines are periodically tested for Mycoplasma contamination using DAPI (4',6-diamidino-2-phenylindole) to stain DNA.

\section{Statistical Analysis}

Metabolite profiling data shown in Figure $1 \mathrm{~h}$ and $\mathrm{i}$ were $\log _{10}$-transformed before further analysis to achieve an approximate normal distribution. Missing values were not imputed for univariate analysis. Mixed model analysis of variance (ANOVA) using $\mathrm{R}$ with package nlme was applied to identify differentially expressed intracellular metabolites in non-ferroptotic (HCC38, HCC1806, HCC1143, HCC70) compared to ferroptotic (MDA-MB-468, MDA-MB-231, BT-549, BT-20, Hs-578T) TNBC cell lines. Our previously reported data set of 155 metabolites including 5-6 replicates per cell line ${ }^{21}$ was taken considering "cell line" as random factor and "ferroptosis response" as categoric factor. ANOVA models was read out concerning t-statistics results comprising estimates, t-values, and p-values. Significance level was set to an $\alpha$-error of $5 \%$. The multiple test problem was addressed by calculating the false discovery rate (FDR) using the Benjamini \& Hochberg method. In general, other statistical comparisons were performed using 
Student's t-test unless noted and the threshold for significance was $\mathrm{p}<0.05$. Data is reported as mean and standard error of the mean of at least 2-3 independent experiments unless otherwise stated. All data points reflect measurements of distinct samples.

\section{Lipidomic analysis}

For Figures $2 \mathrm{~h}$ and $\mathrm{i}$, triplicate samples of MDA-MB-231 cells at $\sim 60 \%$ confluence were incubated with vehicle (methanol), $2 \mu \mathrm{M}$ fer-1, $50 \mu \mathrm{M} \alpha \mathrm{ESA}, 50 \mu \mathrm{M} \alpha \mathrm{ESA}$ and $2 \mu \mathrm{M}$ fer-1, or $250 \mathrm{nM}$ ML162 for 3 hours and then were typsinized, washed twice with phosphate buffered saline (PBS) without magnesium or calcium, and resuspended at a concentration of 1500 cells $/ \mu \mathrm{L}$, and frozen in liquid nitrogen. For tumor samples (Figure 3e), four biological replicate tumor samples per condition were homogenized in PBS without magnesium or calcium using a dounce homogenizer to yield a sample containing $5 \mathrm{mg}$ of tumor (wet weight) / $\mathrm{mL}$ and were then frozen in liquid nitrogen.

Mass spectrometry (MS)-based lipid analysis in Figures 2 and 3e were performed by Lipotype GmbH (Dresden, Germany) as described ${ }^{52}$. Lipids were extracted using a two-step chloroform/methanol procedure ${ }^{53}$. Samples were spiked with internal lipid standard mixture containing: cardiolipin 16:1/15:0/15:0/15:0 (CL), ceramide 18:1;2/17:0 (Cer), diacylglycerol 17:0/17:0 (DAG), hexosylceramide 18:1;2/12:0 (HexCer), lyso-phosphatidate 17:0 (LPA), lyso-phosphatidylcholine 12:0 (LPC), lysophosphatidylethanolamine 17:1 (LPE), lyso-phosphatidylglycerol 17:1 (LPG), lyso-phosphatidylinositol 17:1 (LPI), lyso-phosphatidylserine 17:1 (LPS), phosphatidate 17:0/17:0 (PA), phosphatidylcholine 17:0/17:0 (PC), phosphatidylethanolamine 17:0/17:0 (PE), phosphatidylglycerol 17:0/17:0 (PG), phosphatidylinositol 16:0/16:0 (PI), phosphatidylserine 17:0/17:0 (PS), cholesterol ester 20:0 (CE), sphingomyelin 18:1;2/12:0;0 (SM), triacylglycerol 17:0/17:0/17:0 (TAG). After extraction, the organic phase was transferred to an infusion plate and dried in a speed vacuum concentrator. The dried extract was re-suspended in $7.5 \mathrm{mM}$ ammonium acetate in chloroform/methanol/propanol $(1: 2: 4, \mathrm{~V}: \mathrm{V}: \mathrm{V})$ and the 
second step dry extract was re-suspended in a 33\% ethanol solution of methylamine in chloroform/methanol (0.003:5:1; V:V:V). All liquid handling steps were performed using the Hamilton Robotics STARlet robotic platform. Samples were analyzed by direct infusion on a QExactive mass spectrometer (Thermo Scientific) equipped with a TriVersa NanoMate ion source (Advion Biosciences). Samples were analyzed in both positive and negative ion modes with a resolution of $\mathrm{Rm} / \mathrm{z}=200=280000$ for $\mathrm{MS}$ and $\mathrm{Rm} / \mathrm{z}=200=17500$ for MS-MS experiments, in a single acquisition. MS-MS was triggered by an inclusion list encompassing corresponding MS mass ranges scanned in 1 Da increments (Surma et al. 2015). Both MS and MS-MS data were combined to monitor CE, DAG and TAG ions as ammonium adducts; PC, PC O-, as acetate adducts; and CL, PA, PE, PE O-, PG, PI and PS as deprotonated anions. MS only was used to monitor LPA, LPE, LPE O-, LPI and LPS as deprotonated anions; Cer, HexCer, SM, LPC and LPC O- as acetate adducts. Data were analyzed using lipid identification software based on LipidXplorer ${ }^{54,55}$. Data post-processing and normalization were performed using an in-house developed data management system (Lipotype). Only lipid identifications with a signal-to-noise ratio $>5$, and a signal intensity 5 -fold higher than in corresponding blank samples were considered for further data analysis.

\section{Live Cell Imaging}

For the time lapse imaging, BT-549 cells were seeded in a 6-well plate at 125,000 cells/well. After 24 hours, stauroporine $(500 \mathrm{nM}), \alpha$ ESA $(25 \mu \mathrm{M})$, or ML162 (500 nM) were added along with 2 mM HEPES (pH 7.4) and then each well was overlaid with mineral oil and placed in a temperature-controlled imaging chamber $\left(37^{\circ} \mathrm{C}\right)$. Three fields in each well were imaged every 10 minutes for a 24 -hour period using a Nikon Eclipse TE300 inverted microscope equipped with a Nikon Plan Fluor 10x NA 0.30 objective and a QImaging Retiga EXi camera and using MetaVue software. Movies were assembled using the Fiji implementation of ImageJ ${ }^{56}$. For photomicrographs, cell images were taken using a Nikon Eclipse TE2000 inverted microscope equipped with Nikon Plan Fluor 10x NA 0.30 objective using Metavue (MetaMorph) 
or Ocular (QImaging) software. The micrographs shown in Figure S2b were captured using the EVOS XL Core Imaging System (ThermoFisher Scientific) with the 10x objective.

\section{Detection of lipid peroxidation products}

The Click-iT Lipid Peroxidation Imaging kit (ThermoFisher Scientific) with Alexa Fluor 488 azide was used to detect macromolecules modified with breakdown products from peroxidized lipids according to the recommendations of the manufacturer. Cells were incubated with linoleamide alkyne (LAA) reagent for 16 hours in experiments involving BSO and 4 hours for experiments involving $\alpha$ ESA before being fixed with fixed with $4 \%$ formaldehyde. DAPI was used to visualize DNA. Fluorescent micrographs were captured with a Nikon Eclipse TE2000 inverted microscope with a Nikon Plan Apo 60x A/1.40 Oil using Metavue (MetaMorph) or Ocular (QImaging) software. The fluorescence signal intensity of individual cells was quantified using ImageJ (NIH).

\section{Small interfering RNA}

For siRNA knockdown experiments, cells were transfected (DharmaFECT 1, ThermoFisher Scientific) with ON_TARGETplus SMART pools (ThermoFisher Scientific) targeting individual $A C S L$ and $A L O X$ family members, DAGL1, DAGL2, or a non-targeting siRNA pool (Pool \#1, D-001206-13, ThermoFisher Scientific). Efficiency of mRNA depletion was assessed 72 hours post-transfection using qPCR or western blot in the case of ACSL1 (Proteintech, 13989-1-AP). In experiments to determine suppression of cell toxicity resulting from $\alpha \mathrm{ESA}$ or ML162 by silencing expression of individual $A C S L$ or $A L O X$ genes, doses of $\alpha$ ESA or ML162 were selected such that there was 5-15\% remaining viability in cells transfected with non-targeting siRNA following 24 hours of treatment. 


\section{Quantification of cellular phospholipids}

BT-549 cells were transfected with ACSL1 or non-targeting siRNA 72 hours prior to incubation with 25 $\mu \mathrm{M} \alpha \mathrm{ESA}$ or vehicle (methanol) for 8 hours. After 8 hours, cells ( $\sim 4-6 \times 10^{6}$ per replicate) were trypsinized, collected, washed once with phosphate-buffered saline, and frozen in liquid nitrogen. Triplicate samples were collected for each condition. MS analysis of PLs was performed on an OrbitrapTM FusionTM LumosTM mass spectrometer (ThermoFisher Scientific). Phospholipids were separated on a normal phase column (Luna $3 \mu \mathrm{m}$ Silica (2) $100 \AA$, $150 \times 2.0 \mathrm{~mm}$, (Phenomenex)) at a flow rate of $0.2 \mathrm{~mL} / \mathrm{min}$ on a Dionex Ultimate 3000 HPLC system. The column was maintained at $35{ }^{\circ} \mathrm{C}$. The analysis was performed using gradient solvents (A and B) containing $10 \mathrm{mM}$ ammonium formate. Solvent A contained propanol:hexane:water (285:215:5, v/v/v) and solvent B contained propanol:hexane:water (285:215:40, v/v/v). All solvents were LC/MS grade. The column was eluted for 0-23 min with a linear gradient from 10 $\%$ to $32 \% \mathrm{~B} ; 23-32$ min using a linear gradient of 32-65\% B; 32-35 min with a linear gradient of $65-100$ $\%$ B; 35-62 min held at 100\% B; 62-64 min with a linear gradient from $100 \%$ to $10 \%$ B followed by and equilibration from 64 to $80 \mathrm{~min}$ at $10 \% \mathrm{~B}$. The instrument was operated with ESI probe in negative polarity mode. Analysis of LC/MS data was performed using software package Compound Discoverer (ThermoFisher Scientific) with an in-house generated analysis workflow and oxidized phospholipid database.

\section{Quantitative PCR}

Total RNA was isolated using an RNeasy kit (Qiagen) and tested for quality on a Bioanalyzer (Agilent Technologies). RNA concentrations were determined with a NanoDrop spectrophotometer (ThermoFisher Scientific). RNA was reverse transcribed using Moloney murine leukemia virus reverse transcriptase (Ambion- ThermoFisher Scientific) and a mixture of anchored oligo-dT and random decamers (Integrated DNA Technologies). Two reverse-transcription reactions were performed for each sample using either 100 or $25 \mathrm{ng}$ of input RNA. Aliquots of the cDNA were used to measure the expression levels of the genes with 
the primers, and Power SYBR Green master mix (Applied Biosystems, ThermoFisher Scientific) on a 7900 HT sequence detection system (Applied Biosystems, ThermoFisher Scientific). Cycling conditions were $95^{\circ} \mathrm{C}, 15 \mathrm{~min}$, followed by 40 (two-step) cycles $\left(95^{\circ} \mathrm{C}, 15 \mathrm{~s} ; 60^{\circ} \mathrm{C}, 60 \mathrm{~s}\right) . \mathrm{Ct}$ (cycle threshold) values were converted to quantities (in arbitrary units) using a standard curve (four points, four-fold dilutions) established with a calibrator sample. The primers used were as follows: ACSL1 (GACATTGGAAAATGGTTACCAAATG, GGCTCACTTCGCATGTAGATA), ACSL3 (CGAAGCTGCTATTTCAGCAAG, CTGTCACCAGACCAGTTTCA), ACSL4 (TCTTCTCCGCTTACACTCTCT, CTTATAAATTCTATCCATGATTTCCGGA), ACSL5 (GGAGAATACATTGCACCAGAGA, ACTCCTACTAAGGATGACCGT), and GPX4 (ACGTCAAATTCGATATGTTCAGC, AAGTTCCACTTGATGGCATTTC). 36B4 was used as the normalizer (CCCATTCTATCATCAACGGGTACAA, CAGCAAGTGGGAAGGTGTAATCC).

\section{RNAseq}

Total RNA was isolated using the RNeasy kit (Qiagen) from three independent replicates of MDA-MB231 cells incubated for 5 hours with ML162 $(125 \mathrm{nM})$ and $\alpha \mathrm{ESA}(100 \mu \mathrm{M})$ and 2 replicates for the control incubated with the vehicle for $\alpha$ ESA (methanol). Three biological replicate tung-oil or control MDA-MB231 xenograft tumor samples were homogenized in ice-cold PBS using a dounce homogenizer and total RNA was isolated using the RNeasy kit (Qiagen). The stranded mRNA-seq library was generated from 1000 ng of total RNA from each sample using Truseq stranded mRNA library kit (Illumina) according to the product instructions. In short, mRNAs were enriched twice via poly-T-based RNA purification beads, and subjected to fragmentation at $94{ }^{\circ} \mathrm{C}$ for 8 minutes via the divalent cation method. The first strand cDNA was synthesized by SuperscriptII (ThermoFisher Scientific) and random primers at $42{ }^{\circ} \mathrm{C}$ for 15 mins, followed by second strand synthesis at $16^{\circ} \mathrm{C}$ for 1 hour. During second strand synthesis, the dUTP was used to replace dTTP, thereby the second strand was quenched during amplification. A single 'A' nucleotide is added to the $3^{\prime}$, ends of the blunt fragments at $37^{\circ} \mathrm{C}$ for 30 minutes. Adapters with illuminaP5, P7 sequences as well as indices were ligated to the cDNA fragment at $30{ }^{\circ} \mathrm{C}$ for 10 minutes. After Ampure 
bead (BD) purification (Beckman Coulter), a 15-cycle PCR reaction was used to enrich the fragments. PCR was set at $98^{\circ} \mathrm{C}$ for $10 \mathrm{~s}, 60^{\circ} \mathrm{C}$ for $30 \mathrm{~s}$ and extended at $72{ }^{\circ} \mathrm{C}$ for $30 \mathrm{~s}$. Libraries were again purified using AmPure beads, checked for quality check with a Bioanalyzer (Agilent Technologies) and quantified with Qubit (Invitrogen). Sample libraries were subsequently pooled and loaded to the HiSeq2500 and sequenced using a Hiseq rapid SRcluster kit and HiSeq rapid SBS kit (Illumina). Single 50bp reads were generated for the bioinformatic analysis. Raw sequence reads were aligned to the human genome (hg38) using the Tophat algorithm ${ }^{57}$ and Cufflinks algorithm ${ }^{58}$ was implemented to assemble transcripts and estimate their abundance. Cuffdiff ${ }^{59}$ was used to statistically assess expression changes in quantified genes in different conditions.

\section{Cell viability assays and reagents}

Cells were seeded in 96-well plates (Corning 3917, 3125-6250 cells per well) and treated with compounds 24 hours after plating. Compounds were purchased from Cayman Chemical with the exception of linolenic acid, linoleic acid, docosahexaenoic acid, conjugated linoleic acid (16413), arachidonic acid, Z-VAD-FML, ONO-RS-083, and (+)- $\alpha$-tocopherol (T3634), which were purchased from Sigma Aldrich. RSL3 was purchased from Selleckchem, catalpic acid, $\alpha$-calendic acid, and $\beta$-ESA were obtained from Larodan Fine Chemicals, and punicic acid was purchased from Matreya LLC. Initial aliquots of ferrostatin-1, deferoxamine, and ML162 were generous gifts of the Dixon Lab (Stanford) and additional quantities were purchased from Cayman Chemical. Cell viability was measured using CellTiter-Glo Luminescent Cell Viability Assay (Promega) according to the manufacturer's instructions. Luminescence was measured on an EnSpire Alpha (Perkin Elmer) using the integrated software package. Data were normalized to vehicletreated or sensitizing agent-alone controls and $\mathrm{IC}_{50}$ curves were produced with GraphPad Prism (Version 8). 


\section{Glutathione measurements}

Cellular glutathione was quantified using the GSH/GSSG-Glo kit (Promega) according to the instructions provided by the manufacturer. Drug-treated samples were normalized to parallel cell viability measurements using the CellTiter-Glo assay (Promega).

\section{Quantitation of GPX4 protein levels}

MDA-MB-231 were seeded at a density of 170,000 cells/well of a 6-well plate. After 24 hours,

Cells were incubated for 4 hours with either vehicle (methanol or DMSO) ML162 (200 nM), RSL3 (200nM), erastin $(1 \mu \mathrm{M})$, or $\alpha \operatorname{ESA}(50 \mu \mathrm{M})$. Following treatments, cells were typsinized, pelleted, lysed with RIPA buffer. The western blot was performed using a GPX4 antibody (Abcam, [EPNCIR144], ab125066) (1:1000), $\beta$-actin antibody (Abcam, ab8227) (1:5000) for loading control, and goat anti-rabbit IgG $(\mathrm{H}+\mathrm{L})$ secondary antibody, HRP (ThermoFisher Scientific, 31460) (1:3000). ImageJ (NIH) was used for the densitometric quantification.

\section{Screen of metabolic modulators}

A cell viability screen was performed using a library of 126 small molecule modulators of metabolic pathways (Focus Biomolecules IntelliScreen Cellular Metabolism Library). MDA-MB-231 cells were plated in 384-well format at a density of 1250 cells per well in the presence or absence of $10 \mu \mathrm{M}$ BSO. Compounds ( 5 doses per compound) were delivered by pin transfer 24 hours after cell plating. After 72 hours, cell viability was measured using CellTiter-Glo Luminescent Cell Viability Assay (Promega) according to the manufacturer's instructions. Data were normalized to vehicle-treated controls and were fitted to $\mathrm{IC}_{50}$ curves using GraphPad Prism (Version 8). 


\section{Mouse xenografts}

Orthotopic xenografts were generated by implanting 2.5 million MDA-MB-231 cells in $100 \mu \mathrm{L}$ phosphatebuffered saline (PBS) mixed with $100 \mu \mathrm{L}$ growth factor-reduced Matrigel (Corning) bilaterally into the

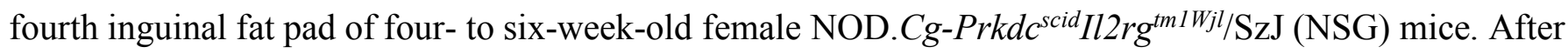
14 days, when tumors were roughly $50 \mathrm{~mm}^{3}$, animals were randomized into treatment groups. Mice were treated on weekdays with either safflower oil (Whole Foods 365) and Tung oil (Sigma-Aldrich 440337) at a dose of $100 \mu \mathrm{L}$ administered by oral gavage. L-BSO (Sigma) was administered via the drinking water (20 $\mathrm{mM}$ ) ad libitum as previously reported ${ }^{21,60}$. The Fox Chase Cancer Center Institutional Animal Care and Use Committee reviewed and approved all animal procedures as compliant with relevant ethical regulations.

\section{Tissue preparation, histology, and immunohistochemistry}

Tumor sections and lung sections were fixed in 10\% formalin for 24-48 hours, dehydrated and embedded in paraffin. Immunohistochemical staining was performed by standard methods. Tissue sections were incubated with primary antibodies to anti-human Ki-67 (Clone MIB-1, DAKO) and cleaved caspase-3 (Cell Signaling; \#9661) at $4{ }^{\circ} \mathrm{C}$ in a humidified chamber overnight. To quantify lung metastases, lungs from three mice per treatment group were fixed, sectioned and hematoxylin and eosin stained. Ten equally sized regions ( 2 from each lobe) were randomly selected and scored manually for area occupied by cancerous tissue. Micrographs were captured with a Nikon DS-Fil camera (Melville, NY, USA) on a Nikon Eclipse $50 \mathrm{i}$ microscope and quantified using a ScanScope CS5 slide scanner (Aperio).

\section{Data Availability}

All data generated or analyzed during this study are included in this published article (and its supplementary information files) or are available from the corresponding author on reasonable request. 


\section{REFERENCES FOR METHODS}

51. Soule, H.D. et al. Isolation and characterization of a spontaneously immortalized human breast epithelial cell line, MCF-10. Cancer Res 50, 6075-86 (1990).

52. Sampaio, J.L. et al. Membrane lipidome of an epithelial cell line. Proc Natl Acad Sci US A 108, 1903-7 (2011).

53. Ejsing, C.S. et al. Global analysis of the yeast lipidome by quantitative shotgun mass spectrometry. Proc Natl Acad Sci U S A 106, 2136-41 (2009).

54. Herzog, R. et al. A novel informatics concept for high-throughput shotgun lipidomics based on the molecular fragmentation query language. Genome Biol 12, R8 (2011).

55. Herzog, R. et al. LipidXplorer: a software for consensual cross-platform lipidomics. PLoS One 7, e29851 (2012)

56. Schindelin, J. et al. Fiji: an open-source platform for biological-image analysis. Nat Methods 9, 67682 (2012).

57. Trapnell, C., Pachter, L. \& Salzberg, S.L. TopHat: discovering splice junctions with RNA-Seq. Bioinformatics 25, 1105-11 (2009).

58. Trapnell, C. et al. Transcript assembly and quantification by RNA-Seq reveals unannotated transcripts and isoform switching during cell differentiation. Nat Biotechnol 28, 511-5 (2010).

59. Trapnell, C. et al. Differential analysis of gene regulation at transcript resolution with RNA-seq. Nat Biotechnol 31, 46-53 (2013).

60. Watanabe, T. et al. A novel model of continuous depletion of glutathione in mice treated with Lbuthionine (S,R)-sulfoximine. J Toxicol Sci 28, 455-69 (2003). 


\section{FIGURE LEGENDS}

Figure 1. Glutathione depletion triggers ferroptosis in a subset of TNBC cell lines and elevated polyunsaturated fatty acid levels are associated with vulnerability to ferroptosis. a, Light micrographs of BT549 cells treated with $10 \mu \mathrm{M}$ BSO for 72 hours in the presence or absence of $20 \mu \mathrm{M} \mathrm{Z-VAD}$ or fer1. Scale bar represents $200 \mu \mathrm{m}$. b, BT-549 cell viability dose-response curves for BSO with or without 20 $\mu \mathrm{M}$ fer-1. Error bars, here and below, denote standard error of the mean. c, Relative viability of BT-549 cells treated with $10 \mu \mathrm{M}$ BSO and the indicated concentration of deferoxamine (DFO) for 72 hours. Values were normalized to account for loss of viability associated with DFO. d, Representative fluorescent micrographs of BT-549 cells with the indicated treatment. Green corresponds to cellular macromolecules modified with breakdown products from peroxidized lipids visualized using the Click-iT lipid peroxidation detection kit (ThermoFisher Scientific). DNA is stained blue. Scale bar represents $20 \mu \mathrm{m}$. e, Quantitation of lipid peroxidation products from individual cells $(n=50$ per condition) in $\mathbf{d}$. Lines represent the mean. f, The $\log _{2}$-transformed ratio of the $\mathrm{IC}_{50}$ of $\mathrm{BSO}$ in the presence or absence of $2 \mu \mathrm{M}$ fer-1 for each cell line. Cell lines in which fer-1 reduced cell death from BSO >8-fold are designated "ferroptotic". g, Box and whiskers plot of the $\log _{2}$-transformed $\mathrm{IC}_{50}$ values for ML162 in non-ferroptotic (turquoise) and ferroptotic (red) TNBC cell lines (as defined in $\mathbf{f}$ ). The line represents the median value and the whiskers show minimum and maximum values. $\mathbf{h}$, Box plots showing the $\log _{10}$-transformed, median-normalized relative levels of phosphatidylcholine (C18:0, C18:2) and $\mathbf{i}$, phosphatidylcholine (C16:0, C18:2) in non-ferroptotic and ferroptotic TNBC cell lines. Each cell line is represented by at least 5 replicates. The line shows the median value, the whiskers represent the upper and lower adjacent values, and outliers are shown as dots.

\section{Figure 2. Conjugated linolenic fatty acids are incorporated into cellular lipids and induce ferroptosis}

in TNBC cells. a, Structure of $\alpha$-eleostearic acid ( $\alpha$ ESA). b, Cell viability dose-response curves for $\alpha$ ESA and the specified additional compound in MDA-MB-231 cells. Cells were treated for 72 hours. Error bars in this and subsequent panels represent standard error of the mean. c, Relative viability of MDA-MB-231 
cells incubated with the indicated doses of $\alpha$ ESA and DFO for 72 hours. Values were normalized to account for loss of viability associated with DFO. d, Quantitation of lipid peroxidation products in individual cells with the specified treatment. The line indicates the mean. From left to right, $\mathrm{n}=42,29,36$, and 14 . * signifies $\mathrm{p}<0.0001$. e, Cell viability dose-response curves for $\alpha$ ESA for three TNBC cell lines (red lines) and non-cancerous MCF-10A controls (black dashed line). f, Relative cell viability for three TNBC cell lines 72 hours after treatment with $\alpha$ ESA or the combination of $\alpha E S A$ and fer-1. g, Schematic representing the location and double bond geometry of 18-carbon conjugated polyunsaturated fatty acids. Cell viability $\mathrm{IC}_{50}$ values are shown for each conjugated PUFA from at least two biological replicate measurements in MDA-MB-231 cells after 72 hours treatment. $\mathbf{h}$, Percent of the mole fraction for each of the indicated classes of lipid that contain 18:3 (18 carbon, 3 double bonds) fatty acids consistent with $\alpha$ ESA. i, Mole percent of each lipid class as a fraction of total lipids in MDA-MB-231 cells incubated with vehicle, $2 \mu \mathrm{M}$ fer-1, $50 \mu \mathrm{M} \alpha \mathrm{ESA}, 50 \mu \mathrm{M} \alpha \mathrm{ESA}$ and $2 \mu \mathrm{M}$ fer-1, or $250 \mathrm{nM}$ ML162 for 3 hours. Error bars in $\mathbf{h}$ and $\mathbf{i}$ show standard deviation. $\mathrm{CE}=$ cholesterol esters, $\mathrm{Cer}=$ ceramide, $\mathrm{DAG}=$ diacylglycerol, Hexcer $=$ hexosylceramide, LPC = lysophosphatidylcholine, LPC O- = ether-linked lysophosphatidylcholine, LPE = lysophosphatidylethanolamine, LPE O- = ether-linked lysophosphatidylethanolamine, LPG = lysophosphatidylglycerol, LPI = lysophosphatidylinositol, $\quad$ LPS = lysophosphatidylserine, PA = phosphatidate, $\mathrm{PC}=$ phosphatidylcholine, $\mathrm{PC} \mathrm{O}-=$ ether-linked phosphatidylcholine, $\mathrm{PE}=$ phosphatidylethanolamine, PE O- = ether-linked phosphatidylethanolamine, $\mathrm{PG}=$ phosphatidylglycerol, PI $=$ phosphatidylinositol, $\mathrm{PS}=$ phosphatidylserine, $\mathrm{SM}=$ sphingomyelin, $\mathrm{TAG}=$ triacylglycerol

Figure 3. Tung oil suppresses TNBC xenograft growth and metastasis with markers of ferroptosis. a, Tumor volumes over time for orthotopic MDA-MB-231 xenografts in NSG mice treated orally with either safflower oil (control) or tung oil ( $\mathrm{n}=6$ mice per group with bilateral tumors). $*$ denote $\mathrm{p}$ values, which are 0.003, 0.00001 , and 0.0003 from left to right. Error bars here and below show standard error of the mean. b, Final tumor masses of individual tumors following 24 days of treatment. Lines represent median values. 
c, Representative hematoxylin and eosin-stained lung sections. Regions containing metastatic TNBC cells are outlined. d, Quantification of the percentage of lung area infiltrated by metastatic cells. The line represents the median value and the whiskers show minimum and maximum values. e, Mole percent of total lipids for DAG 34:3 and TAG 52:7 in tumors treated with tung oil (left) or in MDA-MB-231 cells grown in culture and incubated with $50 \mu \mathrm{M} \alpha \mathrm{ESA}$ for 3 hours (right). The p values were calculated using a onesided Student's t-test, not adjusted for multiple hypothesis testing. f, Proportional Venn diagram showing the overlap in genes with transcript levels that were significantly altered by $>2$-fold $(0.05$ false discovery rate) after a 5-hour incubation with $100 \mu \mathrm{M} \alpha \mathrm{ESA}$ or $125 \mathrm{nM}$ ML-162 or in orthotopic MDA-MB-231 xenograft tumors treated as described above for a. Genes commonly altered in all three data sets are listed.

Figure 4. Mechanistic analysis of $\alpha$ ESA-induced ferroptosis. a, Relative viability (blue) and levels of total glutathione (yellow) in MDA-MB-231 cells after 24 hours of treatment with $20 \mu \mathrm{M} \mathrm{BSO}$, or the specified dose of $\alpha$ ESA. Error bars in this and subsequent panels represent standard error of the mean. $\mathbf{b}$, Densitometric quantification of GPX4 protein levels from western blots of lysates from MDA-MB-231 cells that were treated for 4 hours with the indicated agent. * denotes $\mathrm{p}=0.01$, and $* *$ indicates $\mathrm{p}=0.0002$, $\mathrm{n}=3$ independent experiments. c, Cell viability over time for MDA-MB-231 cells treated as specified. * denote $\mathrm{p}$ values from $\mathrm{n} \geq 3$ independent experiments. $\mathrm{p}$ values from left to right are $0.003,0.0001,0.02$, and 0.008. Bar chart showing fold-change in relative cell viability after 72-hour siRNA knockdown of the specified lipoxygenase followed by 24-hour treatment with either ML-162 (blue) or $\alpha$ ESA (yellow) in d, BT-549 $(\mathrm{n}=3)$ or $\mathbf{e}$, MDA-MB-231 cells $(\mathrm{n} \geq 4) .{ }^{*}$ denote $\mathrm{p}$ values below the significance threshold and greater than 2-fold suppression of cell toxicity. The p values for BT-549 from left to right are 0.004, 0.01, and 0.02 . The $\mathrm{p}$ values for MDA-MB-231 are 0.01 and 0.01 . Cell viability dose-response curves for control and Acs14-deficient Pfal cells treated with f, ML-162 or g, $\alpha$ ESA. h, Bar charts showing fold change in the fraction of viable BT-549 ( $\mathrm{n} \geq 3$ ) or $\mathbf{i}$, MDA-MB-231 cells $(\mathrm{n} \geq 3)$, relative to cells transfected with a non- 
targeting siRNA, 72 hours after transfection with the designated ACSL siRNA followed by 24-hour treatment with either ML-162 (blue) or $\alpha$ ESA (yellow). * signify p values below the significance threshold and greater than 2-fold suppression of cell toxicity. The p values from left to right for BT-549 are 0.005 and 0.01 and for MDA-MB-231 are 0.005, 0.0003, 0.03, 0.03, and 0.04. Complete data for the bar charts in d, $\mathbf{e}, \mathbf{h}$, and $\mathbf{i}$ can be found in Supplementary Table S3.

Figure 5. $\alpha$ ESA treatment increases cellular oxidized phospholipids and a subset of them are decreased by ACSL1 depletion. a, Volcano plot showing $\log _{2}($ fold change $)$ and significance $\left(\log _{10}(1 / \mathrm{p})\right)$ for oxidized phospholipids in BT-549 cells incubated for 8 hours with $25 \mu \mathrm{M} \alpha \mathrm{ESA}$ compared to cell incubated with vehicle (methanol). Each lipid species is colored according to phospholipid class. Abbreviations are as follows: BMP = bis(monoacylglycero)phosphate, $\mathrm{PS}=$ phosphatidylserine, $\mathrm{PE}=$ phosphatidylethanolamine, $\mathrm{PG}=$ phosphatidylglycerol, $\mathrm{PI}=$ phosphatidylinositol, $\mathrm{PC}=$ phosphatidylcholine, $\mathrm{CL}=$ cardiolipin. The arrow indicates a lipid species for which a fold change could not be computed because it was only detected after $\alpha$ ESA treatment. $\mathbf{b}$, Bar charts showing the amounts of di-oxygenated PE species that were significantly increased by $\alpha$ ESA treatment compared to vehicle-treated controls. c, Western blot showing ASCL1 protein in BT-549 cells 72 hours after transfection with either non-targeting siRNA or ACSL1 siRNA. $\beta$-actin is the loading control. Relative amount of all phospholipids quantified that contain 2 acyl chains with 36 carbons and 6 double bonds $(36: 6$, consistent with $\alpha$ ESA incorporation) in $\mathbf{d}$, all phospholipids quantified or e, individual phospholipid classes in BT-549 cells 72 hours after transfection with either non-targeting siRNA or ACSL1 siRNA and then treated with either vehicle or $25 \mu \mathrm{M} \alpha \mathrm{ESA}$ for 8 hours. In e, * indicate significant changes compared to control $(\mathrm{p} \leq 0.001)$ and $* *$ denote significant changes compared to $\alpha$ ESA-treated cells transfected with non-targeting siRNA (significant $p$ values from left to right are $0.00004,0.001,0.02)$. f, Volcano plot showing $\log _{2}$ (fold change) versus $\log _{10}(1 / \mathrm{p}$-value) for oxidized phospholipids in BT-549 cells transfected with ACSL1 siRNA and 
incubated with $25 \mu \mathrm{M} \alpha \mathrm{ESA}$ for 8 hours compared to $\alpha$ ESA-treated cells transfected with non-targeting siRNA. The set is limited to the 51 oxidized phospholipid species that were significantly changed $\alpha$ ESA treatment as shown in $\mathbf{a}$.

Figure 6. Identification of compounds that enhance ferroptosis by $\alpha$ ESA. a, Tumor volumes for orthotopic MDA-MB-231 xenograft tumors in NSG mice treated for 14 days by oral gavage with either 100 $\mu 1$ safflower oil (control, $n=6$ mice, bilateral tumors), tung oil $(\mathrm{n}=4)$, or tung oil and BSO given at $20 \mathrm{mM}$ in drinking water $(\mathrm{n}=4) .{ }^{*}$ denote $\mathrm{p}<0.05$ compared to the control. The $\mathrm{p}$ values from left to right are 0.02 and 0.006 for tung oil-treated mice, and $0.009,0.0001$, and 0.000003 for tung oil and BSO-treated mice. \# indicates $\mathrm{p}=0.004$ compared to tung oil-treated mice. Error bars in this and subsequent panels show standard error of the mean. b, Cell viability dose-response curves for ML162 and the indicated dose of $\alpha$ ESA in BT-549 cells after 72 hours of treatment. $\mathbf{c}$, The $\log _{2}$-transformed ratio of the $\mathrm{IC}_{50}$ of compounds included in a collection of small molecule modulators of cellular metabolism (Focus Biomolecules) in the presence or absence of $10 \mu \mathrm{M}$ BSO in MDA-MB-231 cells treated for 72 hours. $\mathbf{d}$, Relative cell viability in MDA-MB-231 or MCF-10A cells incubated with RHC-80267 alone, with a sensitizing dose of BSO, or with BSO and fer-1. e, Cell viability dose-response curves for RHC-80267 alone, with a minimally lethal, sensitizing dose of $\alpha$ ESA (3-6 $\mu \mathrm{M})$, or with both $\alpha$ ESA and fer-1 in MDA-MB-231 cells. p values generated to assess rescue of cell viability by fer-1 were calculated using a one-sided Student's t-test. f, Relative cell viability in MDA-MB-231 and the specified PLA2 inhibitors (ONO-RS-082 $40 \mu \mathrm{M}, \mathrm{MJ} 335 \mu \mathrm{M}$, CAY10650 $20 \mu \mathrm{M}$ ) with vehicle, a sensitizing dose of $\alpha$ ESA or with $\alpha$ ESA and fer-1. 
a

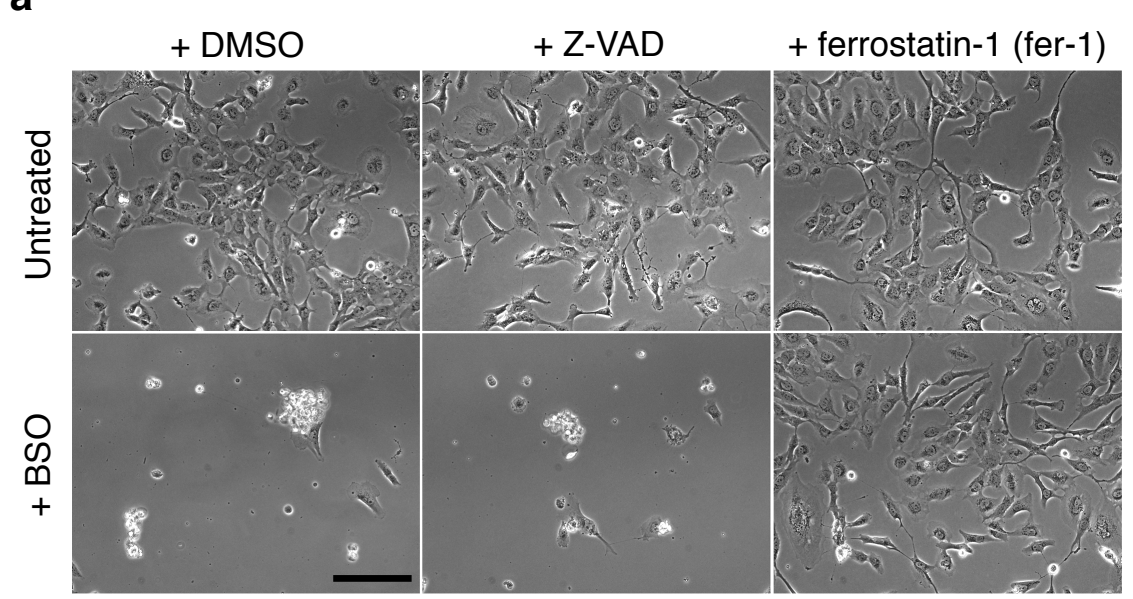

BT-549 (TNBC cell line)

d

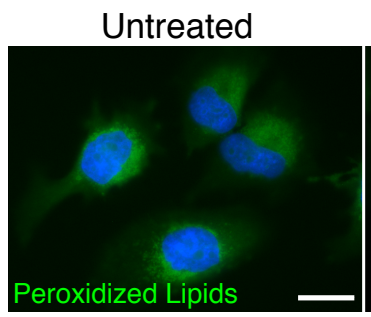

BT-549

+ BSO, fer-1

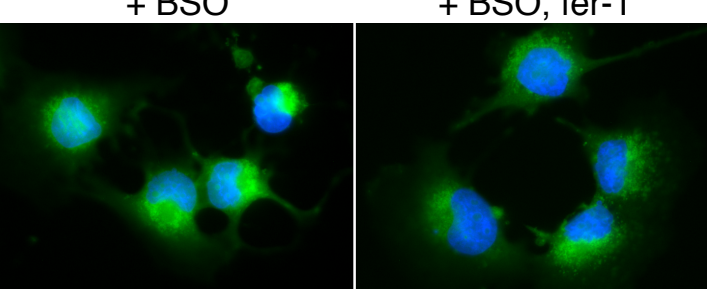

b

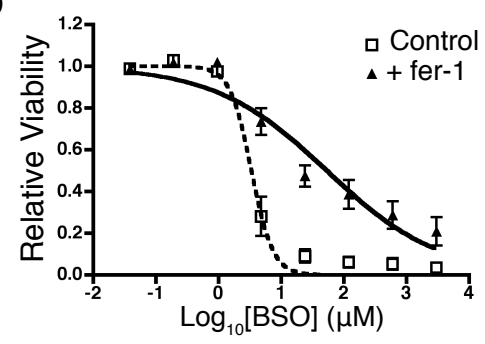

C
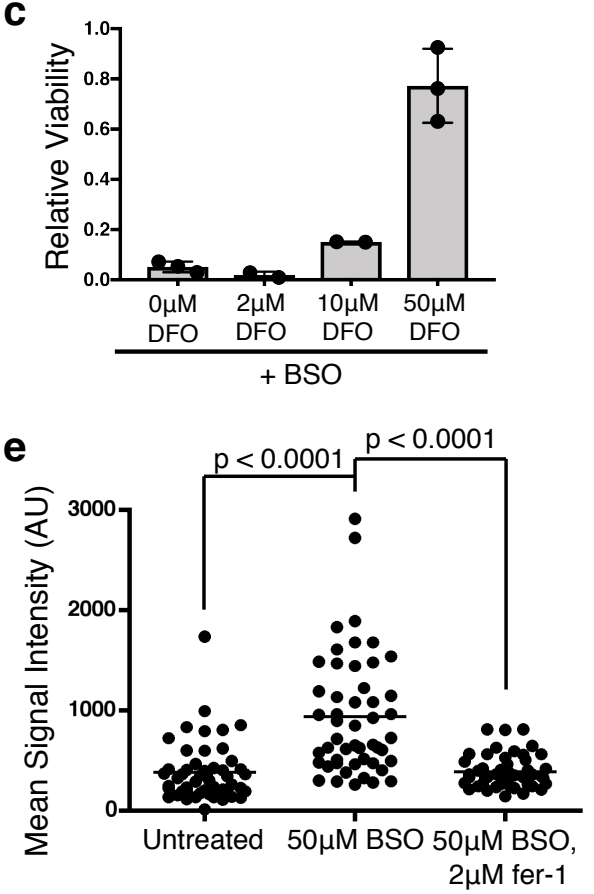

f

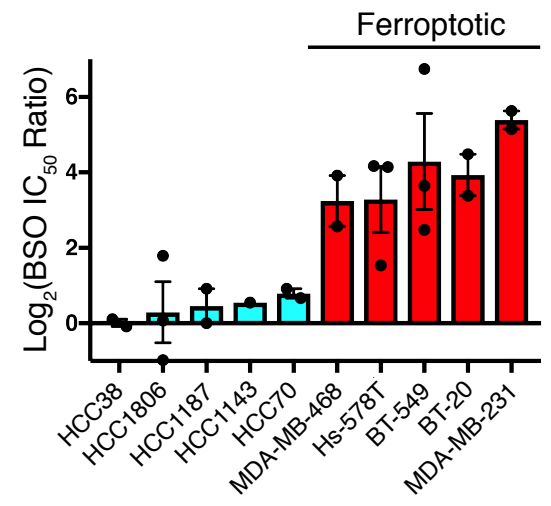

h Phosphatidylcholine (C18:0, C18:2)

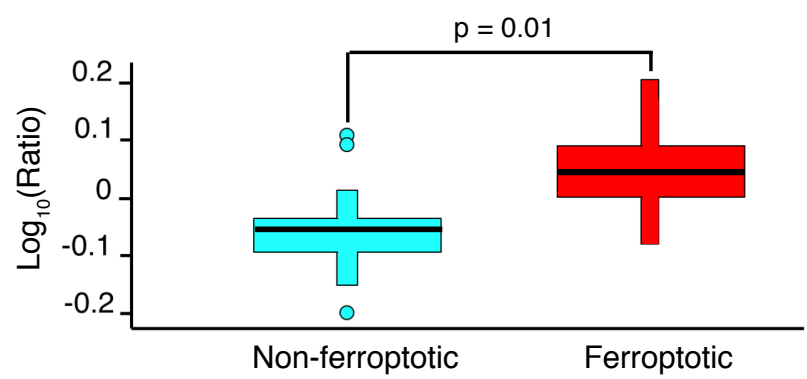

\section{9}

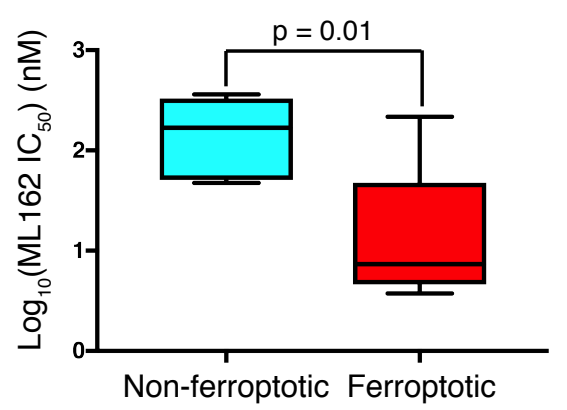

i Phosphatidylcholine (C16:0, C18:2)

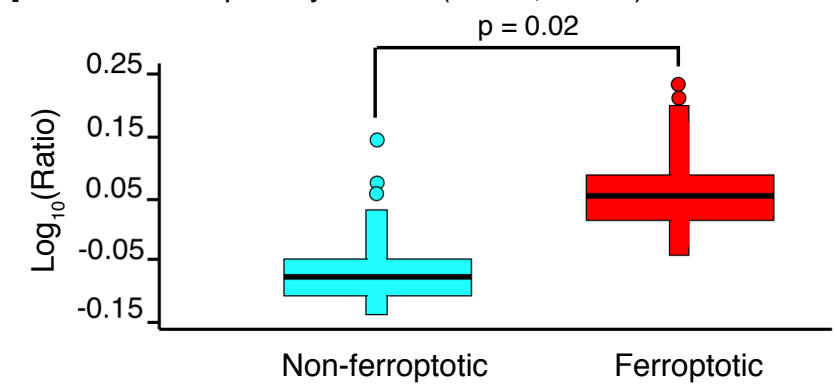


Figure 2ot certified by peer review) is the author/funder, who has granted bioRxiv a license to display the preprint in perpetuity. It is made available

a
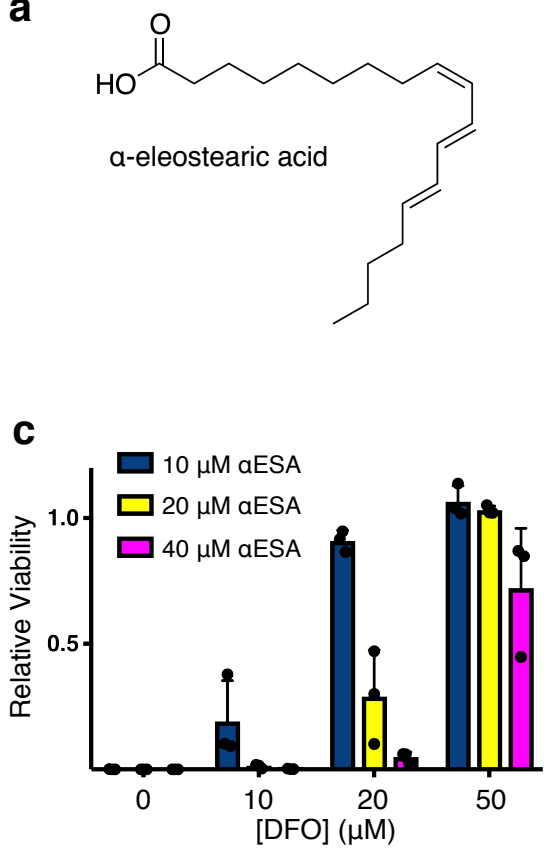

b

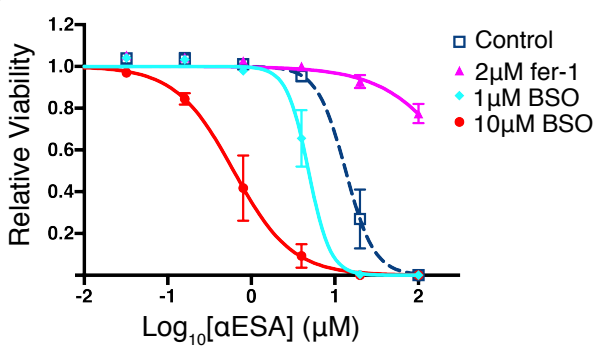

e
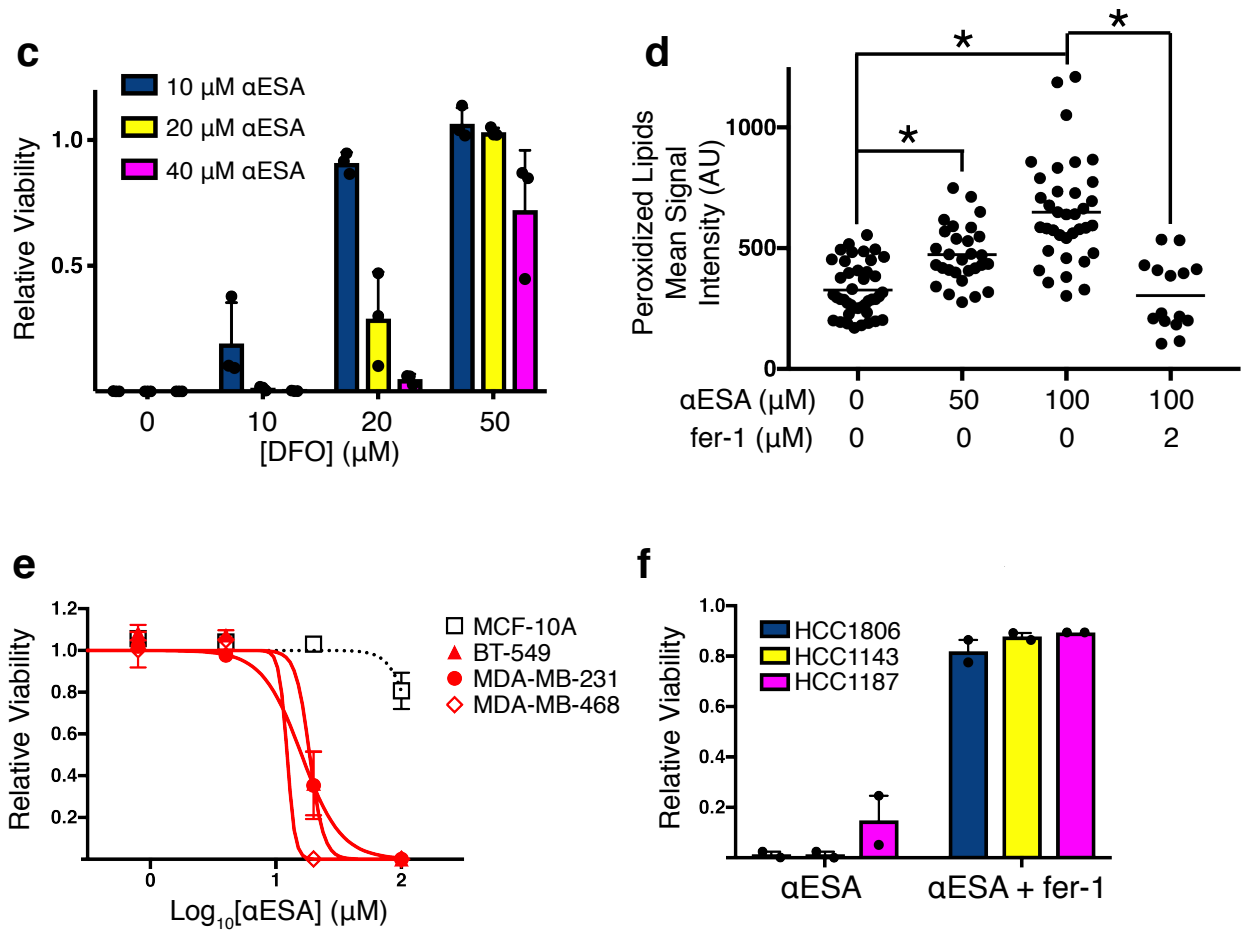

f

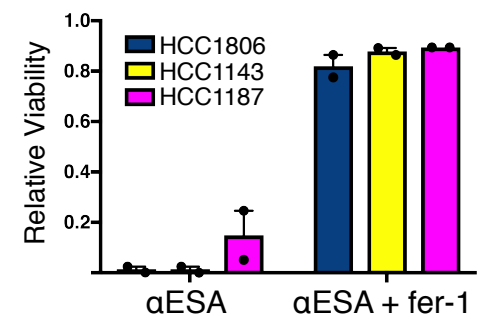

g

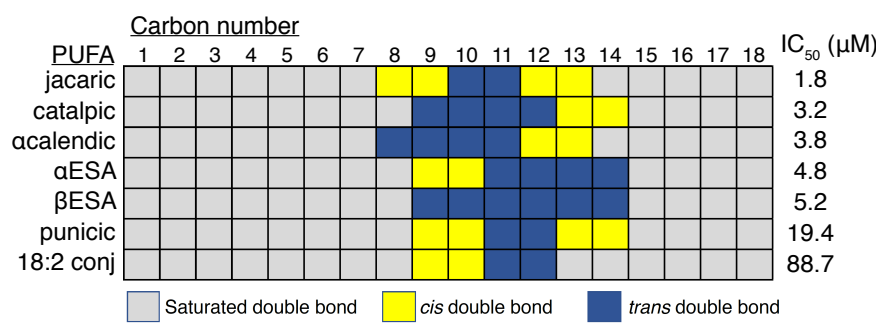

h
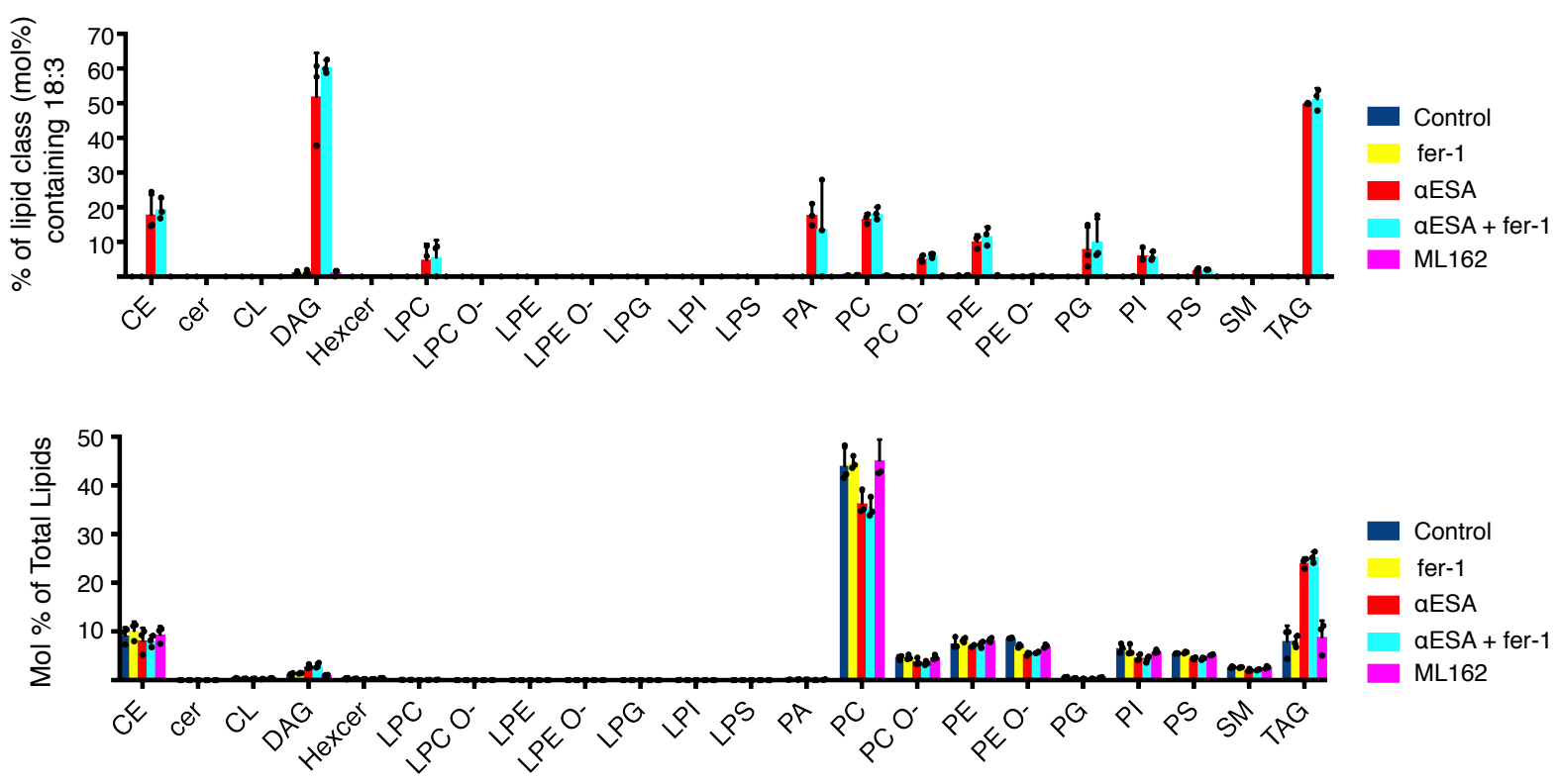
bioRxiv preprint doi: https://doi org/10.1101/556084; this version posted February 20,2019 . The copyright holder for this preprint (which was

Figure Bot certified by peer review) is the author/funder, who has granted bioRxiv a license to display the preprint in perpetuity. It is made available

a

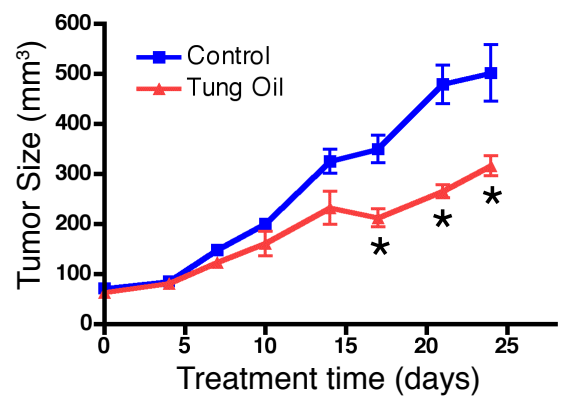

C Control
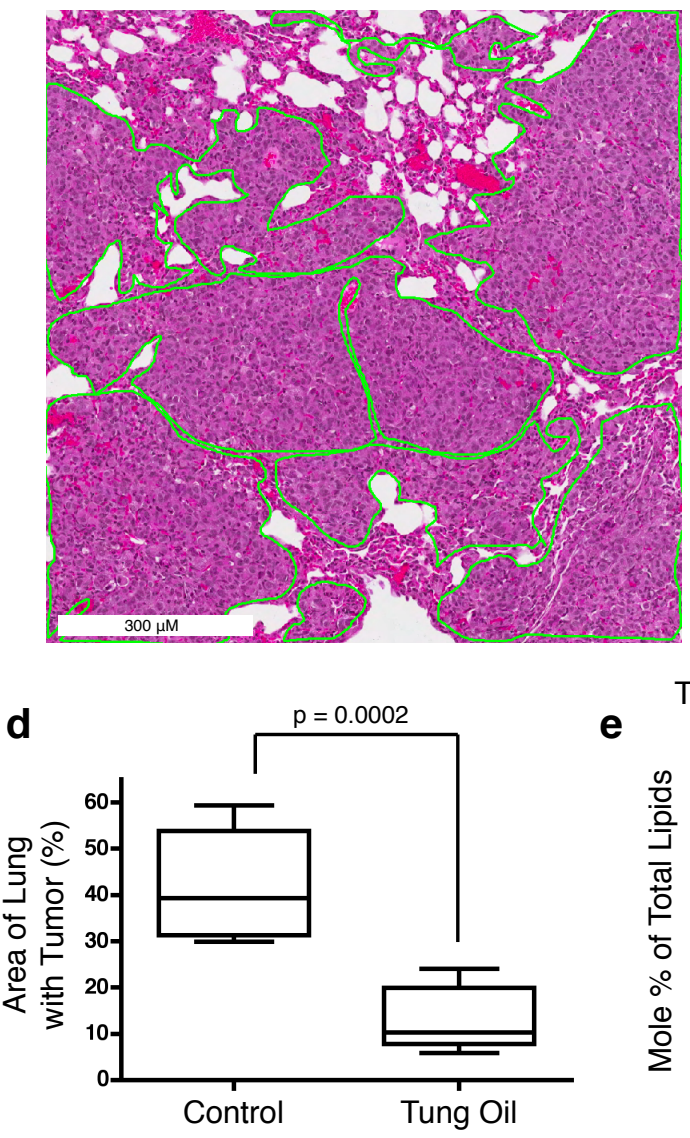

e

Tumor Samples

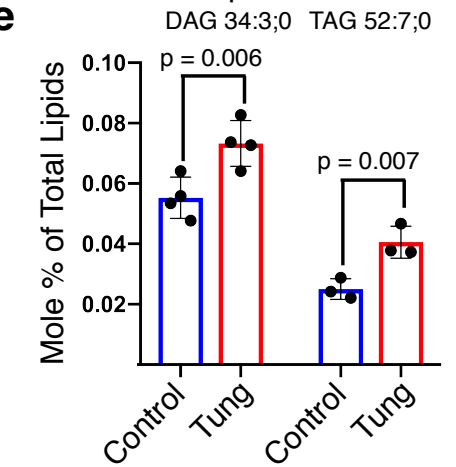

Tung Oil unger aCC-BY-NC-ND 4 . Odntergational license.
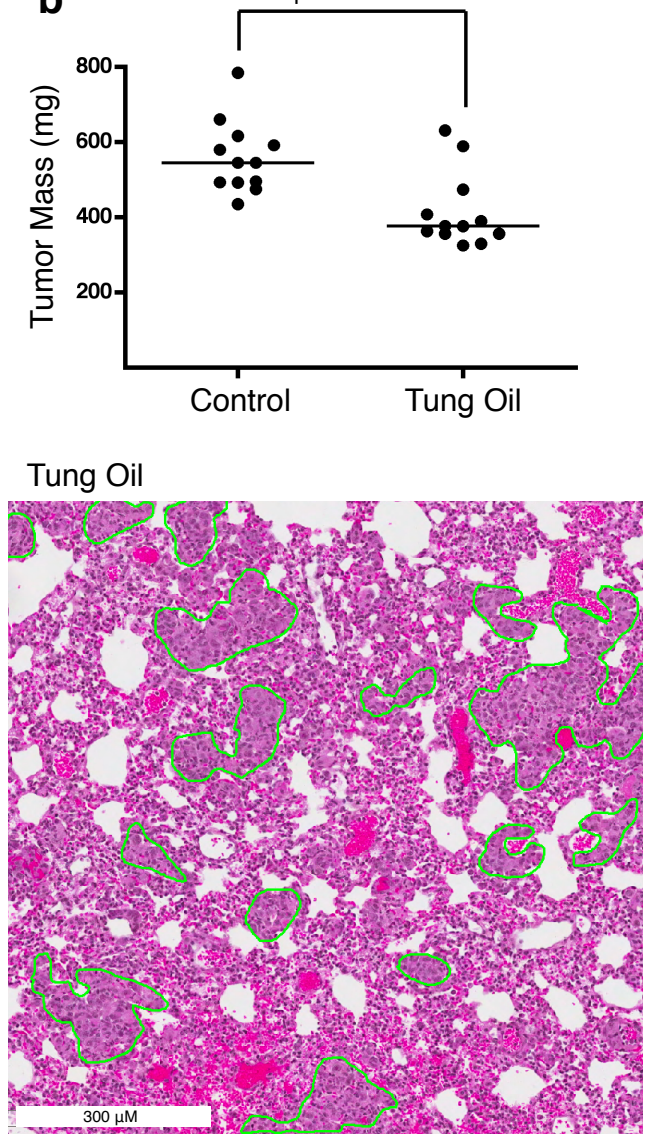

Cell Culture Samples

DAG 34:3;0 TAG 52:7;0

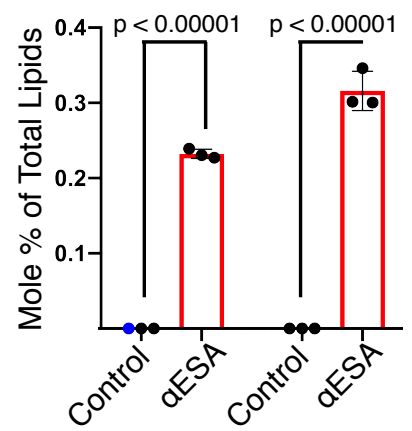

f

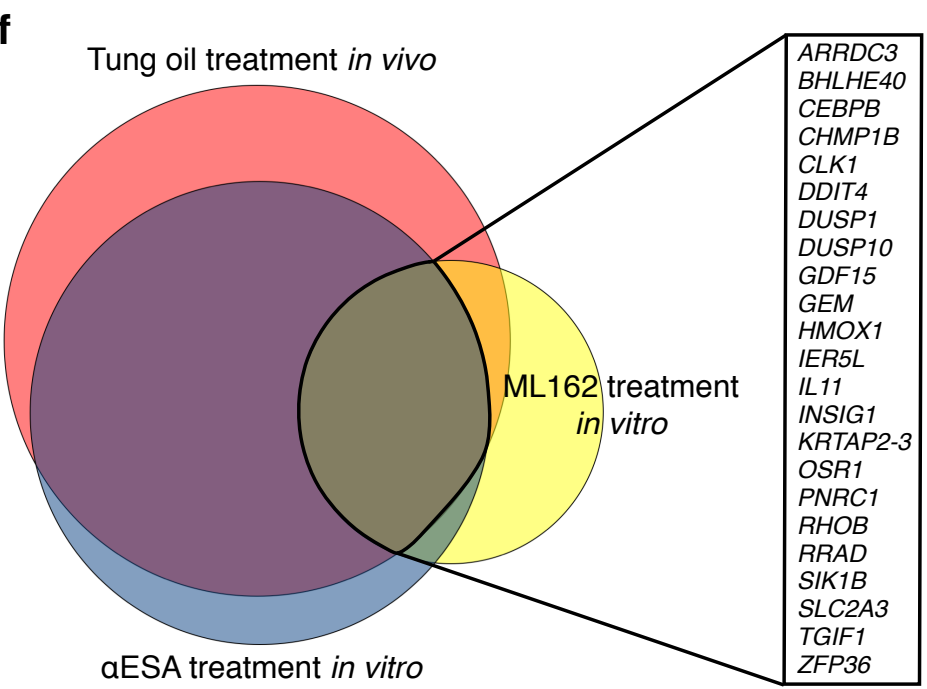


bioRxiv preprint doi: https://doi.org/10.1101/556084; this version posted February 20, 2019. The copyright holder for this preprint (which was Figure 4 ' 4 certified by peer review) is the author/funder, who has granted bioRxiv a license to display the preprint in perpetuity. It is made available
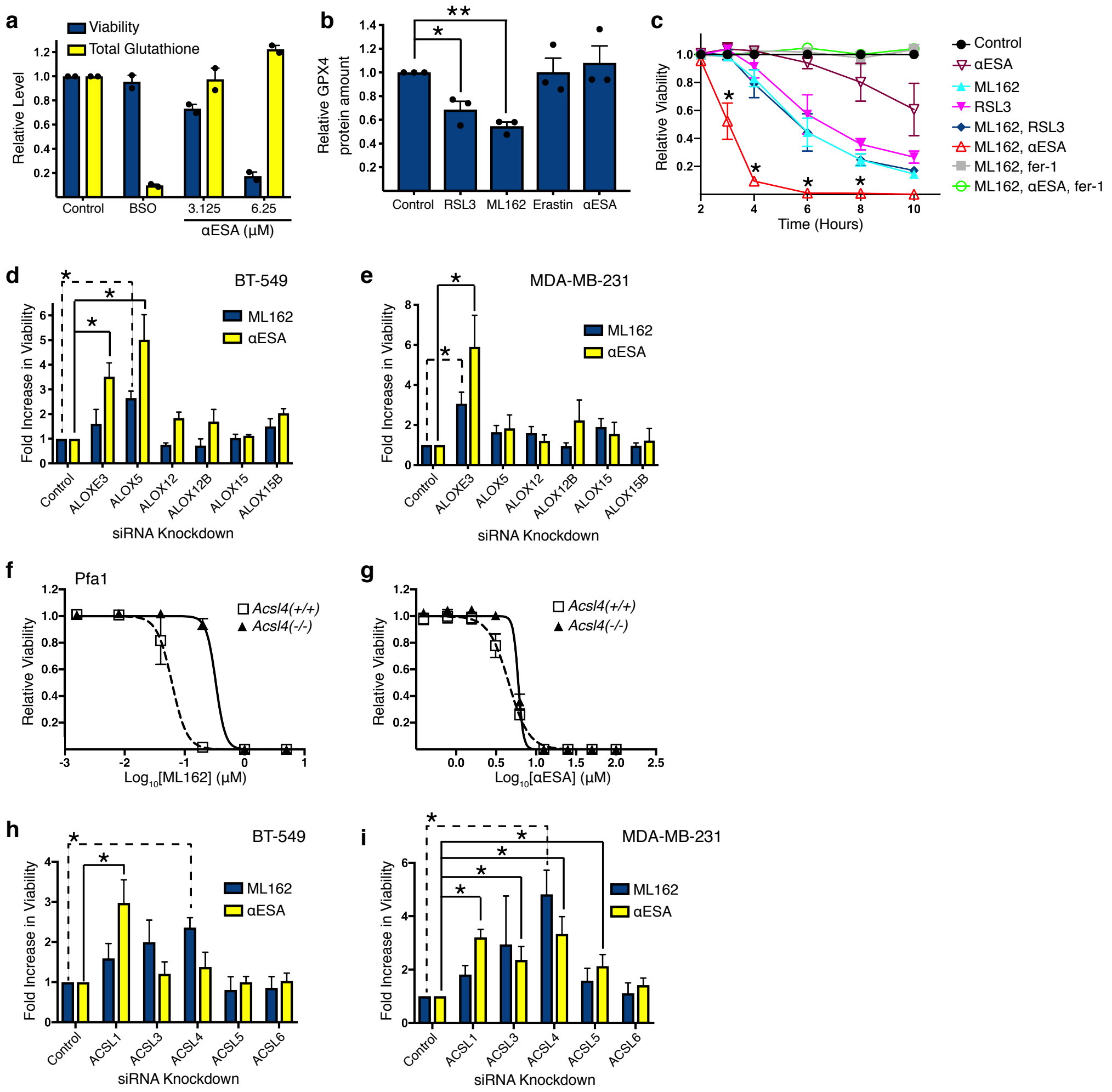
bioRxiv preprint doi: https://doi.org/10.1101/556084; this version posted February 20, 2019. The copyright holder for this preprint (which was

Figure 5 ot certified by peer review) is the author/funder, whohas granted bjoRxiv a license to gisplay the preprint in perpetuity. It is made available

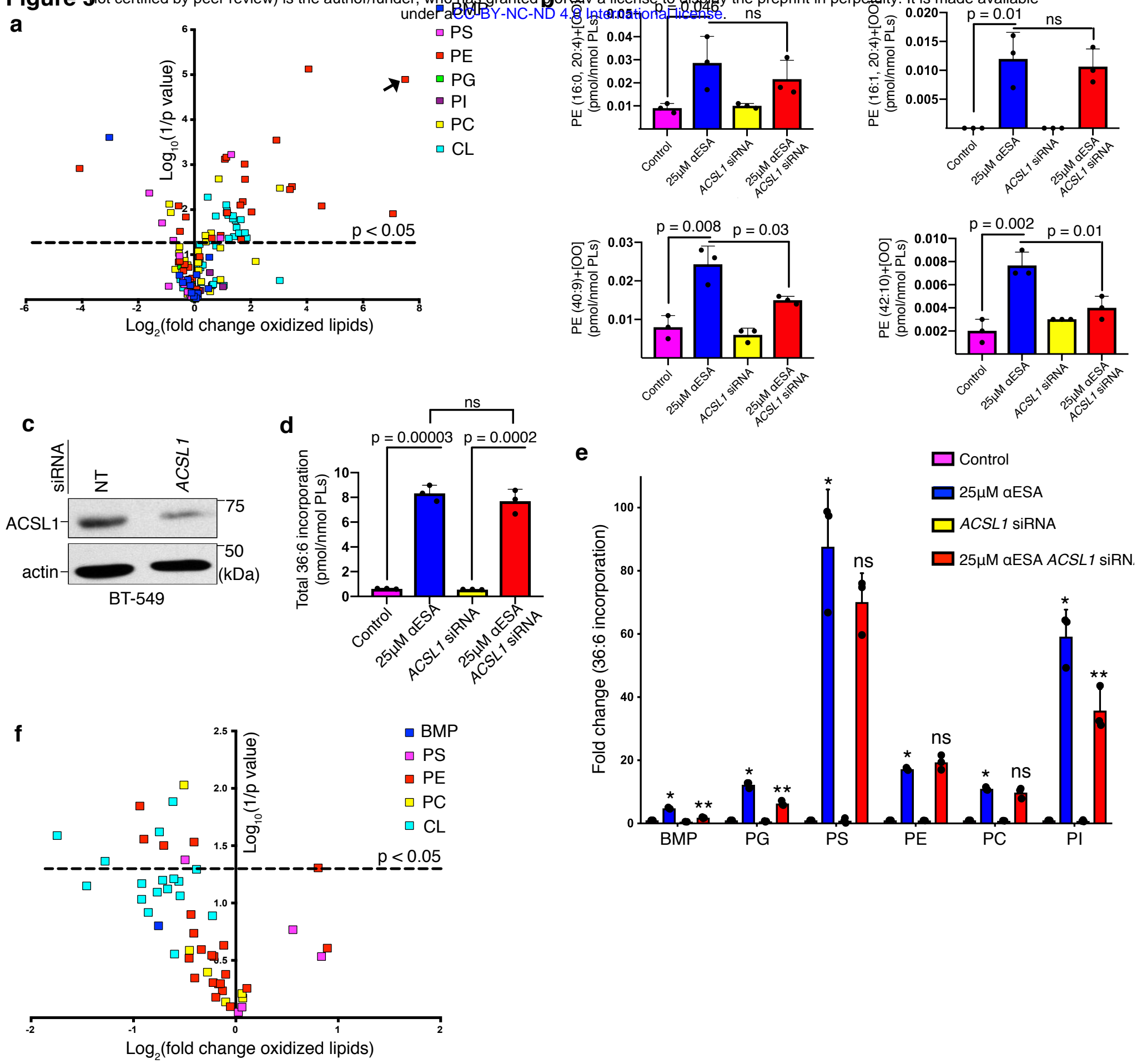


bioRxiv preprint doi: https://doi.org/10.1101/556084; this version posted February 20, 2019. The copyright holder for this preprint (which was Figure ${ }^{\text {n't }}$ certified by peer review) is the author/funder, who has granted bioRxiv a license to display the preprint in perpetuity. It is made available
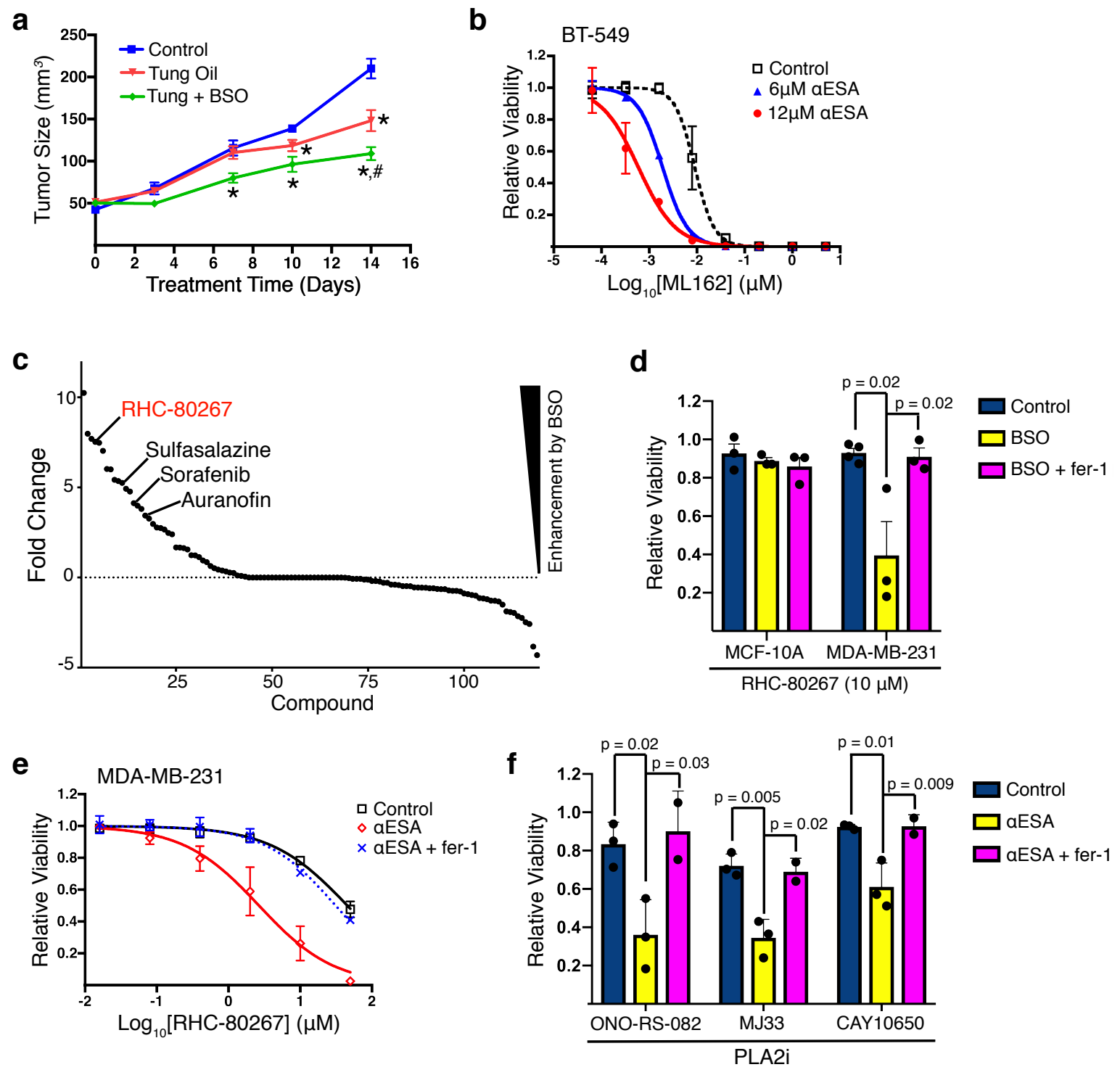